\title{
Subcycle Nonlinear \\ Response of Doped 4H Silicon Carbide Revealed by \\ Two-Dimensional Terahertz Spectroscopy
}

Tarekegne, Abebe Tilahun; Kaltenecker, Korbinian J.; Klarskov, Pernille; Iwaszczuk, Krzysztof; Lu, Weifang; Ou, Haiyan; Norrman, Kion; Jepsen, Peter Und

\section{Published in:}

ACS Photonics

Link to article, DOI:

10.1021/acsphotonics.9b01462

Publication date:

2019

Document Version

Peer reviewed version

Link back to DTU Orbit

Citation (APA):

Tarekegne, A. T., Kaltenecker, K. J., Klarskov, P., Iwaszczuk, K., Lu, W., Ou, H., Norrman, K., \& Jepsen, P. U. (2019). Subcycle Nonlinear Response of Doped 4H Silicon Carbide Revealed by Two-Dimensional Terahertz Spectroscopy. ACS Photonics, 7(1), 221-231. https://doi.org/10.1021/acsphotonics.9b01462

\section{General rights}

Copyright and moral rights for the publications made accessible in the public portal are retained by the authors and/or other copyright owners and it is a condition of accessing publications that users recognise and abide by the legal requirements associated with these rights.

- Users may download and print one copy of any publication from the public portal for the purpose of private study or research.

- You may not further distribute the material or use it for any profit-making activity or commercial gain

- You may freely distribute the URL identifying the publication in the public portal 


\section{Sub-cycle nonlinear response of doped $4 \mathrm{H}$ silicon carbide revealed by two-dimensional terahertz spectroscopy}

Abebe Tilahun Tarekegne, Korbinian J. Kaltenecker, Pernille Klarskov, Krzysztof Iwaszczuk, Weifang Lu, Haiyan Ou, Kion Norrman, and Peter Uhd Jepsen

ACS Photonics, Just Accepted Manuscript • DOI: 10.1021/acsphotonics.9b01462 • Publication Date (Web): 02 Dec 2019

Downloaded from pubs.acs.org on December 9, 2019

\section{Just Accepted}

"Just Accepted" manuscripts have been peer-reviewed and accepted for publication. They are posted online prior to technical editing, formatting for publication and author proofing. The American Chemical Society provides "Just Accepted" as a service to the research community to expedite the dissemination of scientific material as soon as possible after acceptance. "Just Accepted" manuscripts appear in full in PDF format accompanied by an HTML abstract. "Just Accepted" manuscripts have been fully peer reviewed, but should not be considered the official version of record. They are citable by the Digital Object Identifier (DOI®). "Just Accepted" is an optional service offered to authors. Therefore, the "Just Accepted" Web site may not include all articles that will be published in the journal. After a manuscript is technically edited and formatted, it will be removed from the "Just Accepted" Web site and published as an ASAP article. Note that technical editing may introduce minor changes to the manuscript text and/or graphics which could affect content, and all legal disclaimers and ethical guidelines that apply to the journal pertain. ACS cannot be held responsible for errors or consequences arising from the use of information contained in these "Just Accepted" manuscripts. 


\title{
Sub-cycle nonlinear response of doped $4 H$ silicon carbide revealed by two- dimensional terahertz spectroscopy
}

\author{
Abebe T. Tarekegne ${ }^{1}$, Korbinian J. Kaltenecker ${ }^{1}$, Pernille Klarskov ${ }^{2}$, Krzysztof \\ Iwaszczuk ${ }^{1,3}$, Weifang $\mathrm{Lu}^{1}$, Haiyan $\mathrm{Ou}^{1}$, Kion Norrman ${ }^{4}$, and Peter U. Jepsen ${ }^{1, *}$ \\ ${ }^{1}$ DTU Fotonik, Technical University of Denmark, Ørsteds Plads, Building 345, DK-2800 \\ Kongens Lyngby, Denmark \\ ${ }^{2}$ Department of Engineering, Aarhus University, DK-8000 Aarhus C, Denmark \\ ${ }^{3}$ NKT Photonics, Blokken 84, DK-3460 Birkerød, Denmark \\ ${ }^{4}$ Center for Integrative Petroleum Research, King Fahd University of Petroleum and \\ Minerals, KFUPM Box 5070, Dhahran 31261, Saudi Arabia \\ *Corresponding author: puje@fotonik.dtu.dk
}

\begin{abstract}
We investigate single-cycle terahertz (THz) field-induced nonlinear absorption in doped silicon carbide. We find that the nonlinear response is ultrafast, and we observe up to $20 \%$ reduction of transmission of single $\mathrm{THz}$ pulses at peak field strengths of $280 \mathrm{kV} / \mathrm{cm}$. We model the field and temperature dependence of the nonlinear response by finite-difference time-domain simulation that incorporates the temporally nonlocal nonlinear conductivity of the silicon carbide. Nonlinear two-dimensional $\mathrm{THz}$ spectroscopy reveals that the nonlinear absorption has an ultrafast sub-picosecond recovery time, with contributions from both sumfrequency generation and four-wave mixing, in the form of a photon-echo signal. The ultrafast nonlinearity with its equally fast recovery time makes silicon carbide an interesting candidate material for extremely fast nonlinear $\mathrm{THz}$ modulators.
\end{abstract}

\section{Keywords:}


Silicon carbide, nonlinear response, field-driven tunnelling, terahertz spectroscopy, multidimensional spectroscopy, ultrabroadband spectroscopy 


\section{ToC graphics:}

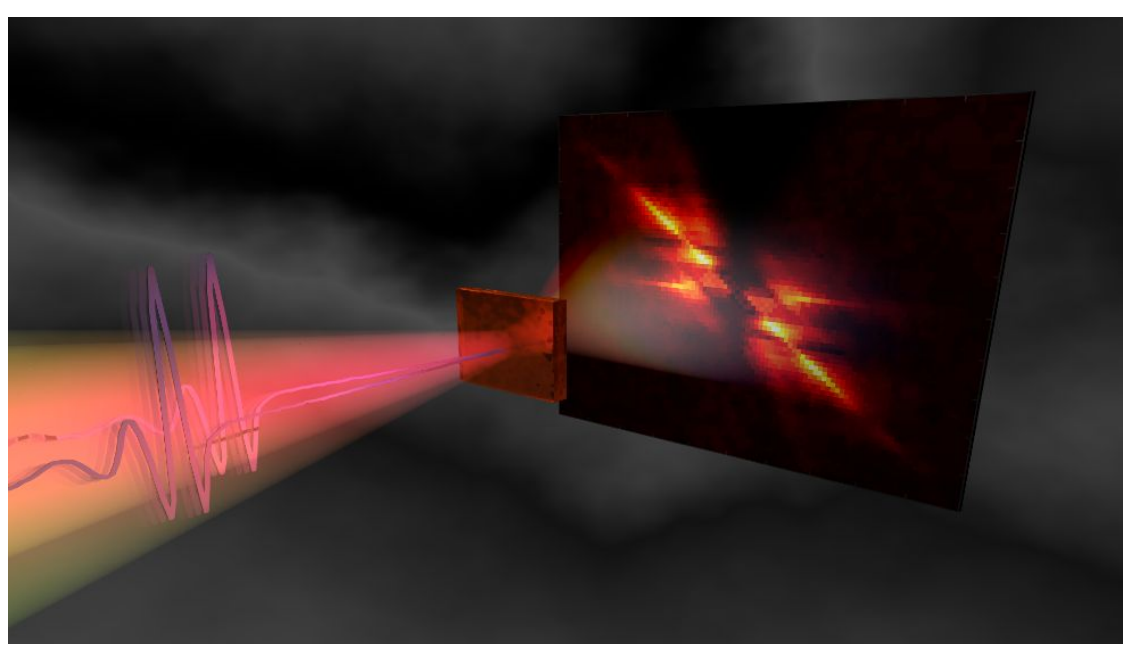


Silicon carbide $(\mathrm{SiC})$ consists of silicon and carbon bilayers arranged in various stacking sequences which results in more than 200 polytypes [1]. SiC has unique features that make it a prominent semiconductor for high power electronic applications including in severe environmental conditions [2, 3]. For example, one of the polytypes, $4 H$-SiC, has three times larger band gap, five times higher breakdown voltage, twice the saturation velocity and three times higher thermal conductivity than silicon $[4,5]$. Challenges in the growth of high quality wafers has previously limited its widespread application in microelectronics and optoelectronics. However, recent improvements in its growth technology has led to commercially availability of high-quality $\mathrm{SiC}$ at wafer-scale, and has reinvigorated interest in the material. The large bandgap of $\mathrm{SiC}$ and its high breakdown voltage makes it one of the most radiation resistant semiconductors, and consequently a fitting material for high power $\mathrm{THz}$ applications.

High-resistivity silicon (HR-Si) is a popular material platform for many $\mathrm{THz}$ systems and devices due to its transparency, high refractive index and low dispersion in the $\mathrm{THz}$ spectral range [6]. Recent advances in THz science and technology enable generation of electric fields in the MV/cm range with table-top systems [7-10]. In strong THz fields, field-driven nonlinear processes such as free carrier generation by impact ionization in doped and even in intrinsic silicon [11], can affect the performance of devices fabricated in silicon. Undoped $\mathrm{SiC}$ has high transparency in the primary $\mathrm{THz}$ spectral range $(0.1-10 \mathrm{THz})$, apart from weak, narrow absorption lines due to weak folded zone phonon modes (at $8 \mathrm{THz}$ in $4 H$-SiC [12]). Due to its large bandgap, SiC response stays linear and transparent to much higher electric fields as compared to silicon, important for $\mathrm{SiC}$ as a material platform for high power $\mathrm{THz}$ applications. Extremely high $\mathrm{THz}$ electric fields are required to induce nonlinear processes in undoped $\mathrm{SiC}$ through interband transition due to its large bandgap energy ( 3.2 eV for $4 H-\mathrm{SiC}$ [13]). However, addition of appropriate dopants introduces energy levels close to the conduction and the valence bands. As we will show here, these states can be ionized by moderate $\mathrm{THz}$ electric fields and release free carriers that induce nonlinearities. In $4 \mathrm{H}$-SiC, two closely spaced conduction subband minima exist at the $M$-symmetry point (see Figure 1(e), $\Delta E=0.12-0.18 \mathrm{eV}$ [14-19]). THz-induced acceleration of free electrons to energies larger than the energetic difference between the two subbands can trigger scattering of electrons into the second subband. Each subband has a distinct curvature in $k$-space, and thus carriers will have distinct effective masses in each subband. Therefore, field-driven scattering of electrons between the subbands will lead to nonlinear transmission of $\mathrm{THz}$ pulses. 
As $\mathrm{THz}$ science and technology matures, nonlinear modulation schemes are extensively investigated in different semiconductors for signal processing applications and expansion of $\mathrm{THz}$ device functionality. For example, THz-induced inter- and intravalley scattering processes in the conduction band have been demonstrated to show $\mathrm{THz}$ absorption bleaching in for instance GaAs, Si, Ge, and InGaAs [18-23]. In line with its potential for high-power $\mathrm{THz}$ applications, the possibility to exploit the nonlinear response in $\mathrm{SiC}$ is beneficial for fabrication of novel integrated nonlinear devices.

Here we demonstrate ultrafast, nonlinear modulation of $\mathrm{THz}$ transmission in doped $4 \mathrm{H}$-SiC which is the most popular polytype due to its high mobility and stability [24, 25]. First, we use ultrabroadband $\mathrm{THz}$ spectroscopy, driven by femtosecond two-color nitrogen plasma generation and air-biased coherent detection (ABCD) for characterization of the linear optical properties of the sample. Then, in single-pulse experiments based on strong $\mathrm{THz}$ fields generated by tilted pulse front excitation of lithium niobate $\left(\mathrm{LiNbO}_{3}\right)$, we observe a $\mathrm{THz}$ induced increase in absorption at strong fields, indicative of an ultrafast nonlinear response. Finally, we use nonlinear two-dimensional $\mathrm{THz}$ spectroscopy based on strong $\mathrm{THz}$ pulse generation by optical rectification in organic crystals to investigate the dynamics of the nonlinear process. We complement the experimental investigation by finite-difference timedomain simulations that include a model for the field-driven carrier dynamics, directly coupled to the propagation of the $\mathrm{THz}$ pulses through $\mathrm{SiC}$.

\section{Ultrabroadband linear optical characterization of the sample}

The investigated sample is a nitrogen, boron and aluminum (Ni-B-Al) co-doped $4 H$-SiC which was grown by physical vapor deposition [26] with the $c$-axis normal to the surface. The sample thickness was measured to be $(230 \pm 10) \mu \mathrm{m}$ by a standard micrometer caliper. The dopant concentration was determined by time-of-flight secondary ion mass spectrometry (TOF-SIMS) based on an ion implanted reference sample. The dopant concentrations near the surface were found to be $(3.9 \pm 0.2) \times 10^{18},(1.1 \pm 0.2) \times 10^{19}$, and $(2.9 \pm 0.2) \times 10^{15} \mathrm{~cm}^{-3}$ for nitrogen, boron and aluminum dopants, respectively. Nitrogen is a donor dopant that introduces energy states close to the conduction band. Aluminum and boron acts as acceptors and introduce energy states in the lower half of the bandgap.

The ionization energy of $\mathrm{N}$ dopant states in $4 \mathrm{H}$-SiC varies in literature, $E_{d h}: 41-66 \mathrm{meV}$ and $E_{d k}: 80-132 \mathrm{meV}$ for the hexagonal $(h)$ and cubic $(k)$ inequivalent lattice sites, respectively [27-30]. The ionization energies of $\mathrm{Al}$ and $\mathrm{B}$ acceptors also vary in the literature, with values 
of $191-230 \mathrm{meV}$ and $285-390 \mathrm{meV}$ for aluminum and boron, respectively [29, 31-33]. Photoluminescence measurements by Sun et al. identify an additional deep B dopant state with binding energy of $540-730 \mathrm{meV}[31,34]$.

Figure 1 shows an overview of the linear optical properties of the sample at room temperature, as recorded by ultrabroadband $\mathrm{THz}$ spectroscopy based on femtosecond two-color $\mathrm{THz}$ generation in a nitrogen plasma [35-38] and detection by air-biased coherent detection (ABCD) $[39,40]$. The experimental methods for this measurement are described in [12], where the linear optical properties of undoped $4 H$ and $6 H \mathrm{SiC}$ were investigated. Figure 1(a) and Figure 1(b) show the temporal waveforms and corresponding frequency spectra of the reference signal (blue curves) recorded without the sample in the beam path, and the sample signal (red curves), recorded after transmission through the $228-\mu \mathrm{m}$ thick sample. The shaded spectral areas indicate the regions of the spectrum where the dynamic range of the measurement is too small for high-quality spectroscopy [41] and thus, we report data in the 1-15 THz range. Based on the amplitude (Figure 1(b)) and phase (not shown) of the sample and reference spectra, we extract the permittivity of the sample [42] as shown in Figure 1(c), where the blue and red curves represent the real and imaginary part of the permittivity, respectively. For comparison, we have included corresponding data for undoped $4 H \mathrm{SiC}[12]$, represented by dotted curves.
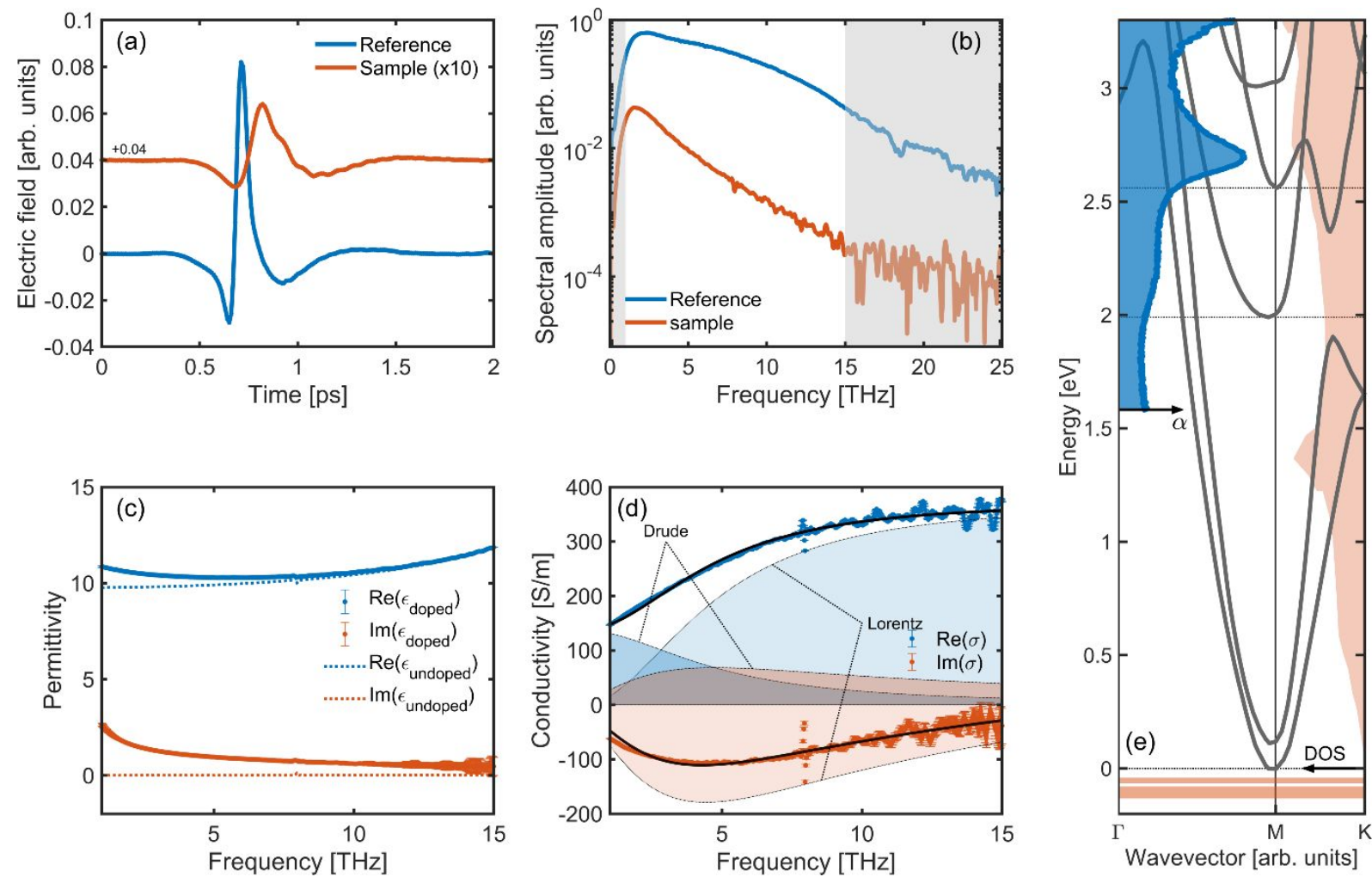

Figure 1. THz-time-domain spectroscopy of co-doped 4H-SiC. (a) Time-domain traces of the reference pulse (blue curve) and sample pulse (red curve). (b) Frequency spectra of the reference and sample signals. (c) Extracted permittivity of the sample, shown together with the permittivity of 
undoped $4 H$-SiC [12]. (d) The extracted conductivity of charge carriers in the co-doped $4 H$-SiC sample. The full, black curves represent the best fit to the data by a Drude-Lorentz model, and the shaded areas indicate the contributions from the Drude and Lorentz terms, respectively. Light blue and red shadings indicate the real and imaginary parts of the contributions, respectively. (e) Our measured optical absorption in the visible range (blue, shaded curve), shown together with the structure of the five lowest conduction bands (solid curves) and density of states (shaded, red curve) (band structure adapted from Ref. [16]), and the location of the dopant energy levels (horizontal, shaded bars).

The general increase in the real part of the permittivity towards high frequencies is due to the strong dispersion of the $\mathrm{TO}$ phonon in $\mathrm{SiC}$ at approximately $25 \mathrm{THz}$. Apart from this, we see that both the real and imaginary parts of the permittivity of the doped sample is higher than for the undoped sample. This difference is due to the optical conductivity of charges in the doped sample. Based on the fundamental relation between permittivity and conductivity ( $\left.\varepsilon(\omega)=\varepsilon_{\infty}+i \sigma(\omega) / \varepsilon_{0} \omega\right)$ the conductivity of the charge carriers is extracted as $\sigma(\omega)=-i \varepsilon_{0} \omega\left(\varepsilon_{\text {doped }}(\omega)-\varepsilon_{\text {undoped }}(\omega)\right)$, where $\varepsilon_{0}$ is the vacuum permittivity and $\omega$ is the angular frequency. In this manner, the strong optical response of the TO phonon, already included in the permittivity function of the undoped sample, is decoupled from the conductivity spectrum in Figure 1(d). The real and imaginary parts of the conductivity are shown in Figure 1(d) as blue and red symbols. Error bars indicate the standard deviation from the mean of a series of repetitive measurement series. The black, solid curves indicate the best fit to the conductivity spectrum based on a Drude-Lorentz model of the form

$$
\sigma(\omega)=\sigma_{\text {Drude }}(\omega)+\sigma_{\text {Lorentz }}(\omega)=\frac{\sigma_{d c}}{1-i \omega \tau_{s}}+\frac{A \omega}{\omega \gamma+i\left(\omega_{0}^{2}-\omega^{2}\right)}
$$

The Drude term with DC conductivity $\sigma_{d c}$ and scattering time $\tau$ represents the conductivity of quasi-free electrons in the conduction band, and the Lorentz term with strength $A$, resonance frequency $\omega_{0}$ and damping rate $\gamma$ represents the conductivity of bound electrons, interpreted here as electrons still bound to the shallow $\mathrm{N}$ dopant sites. The best-fit parameters are $\sigma_{d c}=137 \mathrm{~S} / \mathrm{m}, \quad \tau_{s}=34 \mathrm{fs}, \quad A=3.1 \cdot 10^{5} \mathrm{~S} /(\mathrm{ps} \cdot \mathrm{m}), \quad \omega_{0}=159 \mathrm{ps}^{-1} \mathrm{~A} 104 \mathrm{meV}, \quad$ and $\gamma=879 \mathrm{ps}^{-1}$ A $574 \mathrm{meV}$. The relative contributions from the Drude and Lorentz terms are indicated by the shaded areas in Figure 1(d). The resonance energy of the Lorentz term is comparable to the reported binding energies of the $\mathrm{N}$ dopants as well as the inter-conduction band transition between the two lowest conduction bands (see above), and we therefore attribute the Lorentz part of the conductivity to these two mechanisms. The very broad resonance 
(damping rate is more than five times larger than the resonance frequency) indicate a very short lifetime of the transition.

Based on the DC conductivity and Drude scattering time from the fit, and from the known effective transverse mass of electrons in the conduction band of $\operatorname{SiC}\left(m^{*}=0.43 m_{e}[16]\right)$, we can estimate the concentration of quasi-free electrons in the conduction band to be $n_{0}=\sigma_{d c} m^{*} / e^{2} \tau=6.1 \cdot 10^{16} \mathrm{~cm}^{-3}$. Using the simple Boltzmann relation $n_{0} \approx N_{D} \exp \left(-E_{D} / k_{B} T\right)$ with the fitted 104-meV binding energy at room temperature, we see that approximately $1.8 \%$ of the dopant atoms are ionized, corresponding to $N_{D} \approx 3.3 \cdot 10^{18} \mathrm{~cm}^{-3}$, in good agreement with the ToF-SIM measurements.

This analysis shows that low transmission of the broadband $\mathrm{THz}$ radiation is caused by free electrons in the conduction band and bound electrons at the donor states. This has further been confirmed by optical absorption measurement of the sample in the visible spectral range as shown in the inset of Figure 1(e). The peaks of the absorption coefficient near $2.2 \mathrm{eV}$ and 2.7 $\mathrm{eV}$ are caused by electronic transitions from the conduction band minimum (or $\mathrm{N}$ dopant states) to the higher conduction band states. The much higher binding energy of B dopants than those of $\mathrm{N}$ and the three orders of magnitude lower density of $\mathrm{Al}$ dopants corroborate that $\mathrm{N}$ is the major contributor of free carriers, in the form of conduction band electrons.

\section{Experimental setups for nonlinear single-pulse and two-dimensional spectroscopy}

Two different $\mathrm{THz}$ experimental platforms are used in the nonlinear investigations reported here. Both experiments are driven by amplified laser pulses (SpectraPhysics Solstice Ace, pulse energy of $6 \mathrm{~mJ}$, central wavelength $800 \mathrm{~nm}$, repetition rate $f_{0}=1 \mathrm{kHz}$, pulse width $100 \mathrm{fs}$. We use a standard strong-field $\mathrm{THz}$ generation and detection setup for single-pulse experiments, and a two-pulse experiment for 2D nonlinear $\mathrm{THz}$ spectroscopy. In the one-pulse experiment an intense $\mathrm{THz}$ pulse is generated by optical rectification in a lithium niobate $\left(\mathrm{LiNbO}_{3}\right)$ crystal in the standard tilted-pulse front scheme [7,43]. We detect the temporal shape of the THz pulses by free-space electro-optic sampling [44-46] in a 1-mm thick ZnTe crystal with a balanced detection system locked to the frequency $f_{0} / 2$ of an optical chopper in the generation beam. We use a standard configuration of four off-axis paraboloidal mirrors with the sample placed in the primary focal plane of this $\mathrm{THz}$ beam path, and detection performed in a less tight focus after attenuation of the $\mathrm{THz}$ beam with $\mathrm{HR}-\mathrm{Si}$ wafers, to ensure linearity of the detection. For temperature-dependent measurements, the sample is placed in a closed-cycle cryostat. The field-dependent $\mathrm{THz}$ transmission of the sample is measured by varying the incident field 
strength by relocating a variable number the HR-Si wafers in the collimated $\mathrm{THz}$ beam path either before or after the sample. In this manner, any nonlinearity of the sample is detected as a change in detected signal strength when placing the attenuating wafers before or after the sample. The attenuation of the directly transmitted THz field is $\left(4 n_{S i} /\left(1+n_{S i}\right)\right)^{N}=0.7^{N}$ for $N$ wafers in the beam path [47]. In the collimated beam path the field strength is much lower than in the focus region, and we have confirmed that any nonlinear processes in the HR-Si wafers such as impact ionization [48] are undetectable. The absolute field strength is calibrated by measuring the phase retardation in the electro-optic sampling and relating this to the field strength via the known electro-optic coefficient of ZnTe. Thus, the stated field strengths are minimum values.

Our setup for nonlinear two-dimensional $\mathrm{THz}$ spectroscopy operates by the same principles as described first by Kuehn et al. [49]. In their description of nonlinear two-dimensional $\mathrm{THz}$ spectroscopy, two THz pulses of approximately equal field strength are transmitted through the sample in a collinear manner, separated by a variable pump delay $\tau$. In our implementation we separate the two THz pulses spatially, so that they are incident on the sample at a small angle $\theta \approx 5^{\circ}$. In this manner, we have access to the full field strength of each signal since no beamsplitter is needed for beam overlap. Inside the sample, the propagation angle is $\pm 1.6^{\circ}$ due to the refractive index of the sample. Compared to strictly collinear propagation, this small angle leads to a phase walk-off of $1.5 \mathrm{mrad}$ per wavelength, so in our thin sample the collinear approximation is still valid.

At each pump delay we record the transmitted signal when both pulses $(A+B)$ are present, when only pulse A and B are present, respectively, and determine the two-dimensional temporal map of the nonlinear interaction as

$$
E_{N L}(t, \tau)=E_{A B}(t, \tau)-E_{A}(t, \tau)-E_{B}(t, \tau)
$$

Figure 2(a) illustrates the optical setup used for our implementation of nonlinear 2D $\mathrm{THz}$ spectroscopy [50]. Femtosecond pulses from the Ti:sapphire amplifier, here operating at a repetition rate $f_{0}=500 \mathrm{~Hz}$, are converted to sub-100 fs pulses with a central wavelength of $1450 \mathrm{~nm}$ and pulse energy of $1 \mathrm{~mJ}$ in an optical parametric amplifier (OPA, SpectraPhysics/Light Conversion TOPAS HE). These pulses are split in two equally intense beams, and used for THz generation in organic nonlinear crystals (DSTMS for pulse A and DAST for pulse B, thickness $0.4 \mathrm{~mm}$, from Swiss Terahertz llc). We use separate crystals for generation of the two THz pulses in order to ensure that any nonlinearities detected during the 
temporal overlap of the two pulses originate from the sample and not any unforeseen higherorder nonlineary in the generation process, since each crystal is optically excited to a level quite close to the damage threshold. The two THz beams are collimated by 2" effective focal length (EFL) off-axis parabolic mirrors (OPM) and focused at a small angle with respect to each other by an OPM (EFL 2") on the sample. After the sample, the two beams are focused to a common spot on a GaP electro-optic detection crystal by an OPM with 4" EFL. The delay between the two THz pulses is set by delay stage (DL) 1 , and the temporal profile of the pulses is recorded by electro-optic sampling while scanning DL 2. The simultaneous recording of the signals $E_{A B}, E_{A}, E_{B}$ is obtained by a combination of three optical choppers (CH1-3), all locked to the laser repetition rate as indicated in Figure 2(a). In this manner, 8 subsequent pulses from the laser are used to record the three signals, with a fundamental frequency of $f_{0} / 8$ - here 62.5 Hz. In order to reduce noise in the detection system, we condition the signals from the balanced electro-optic detection with boxcar integrators triggered synchronously at $f_{0} / 4(125 \mathrm{~Hz})$, with delayed gates (0, 2 and $4 \mathrm{~ms}$, respectively, for detection of $\mathrm{AB}, \mathrm{B}, \mathrm{A}$ signals). The signal from each boxcar is then recorded by separate lock-in amplifiers, locked to $f_{0} / 8$.

The peak THz field strength incident on the sample can be adjusted by shifting a suitable number of HR-Si wafers between the parallel sections of the beam before (attenuated) and after (not attenuated) the sample. A larger number of wafers $(N=6)$ was permanently inserted in the $\mathrm{THz}$ beam in the parallel section of the beam after the sample to ensure linearity of the detection. Figure 2(b) shows a temporal scan of the two pulses at delay time $\tau=-4.5 \mathrm{ps}$. The optical power on the crystals is adjusted to yield approximately the same peak field strength of the two pulses. The energy of each pulse is 4.4 and $4.5 \mu \mathrm{J}$ (measured with a Gentec T-Rad pyroelectric detector), and the two THz beams are focused to a common spot size of $230 \mu \mathrm{m}$ at the position of the sample, yielding a focused peak field strength of approximately $2 \mathrm{MV} / \mathrm{cm}$. The spectra of the two pulses peak at $2 \mathrm{THz}$ and extends from 0.3 to $4 \mathrm{THz}(10 \%$ limits), as shown in Figure 2(b). The dip at $1 \mathrm{THz}$ in the spectrum of pulse B is due to self-absorption in DAST. 
(b)

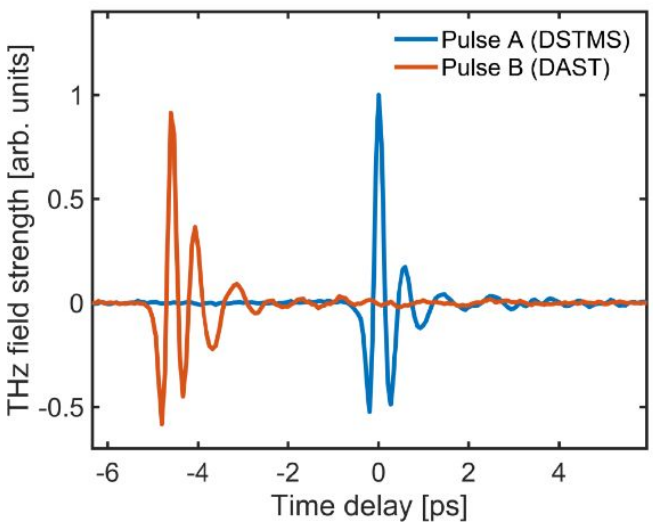

(a)
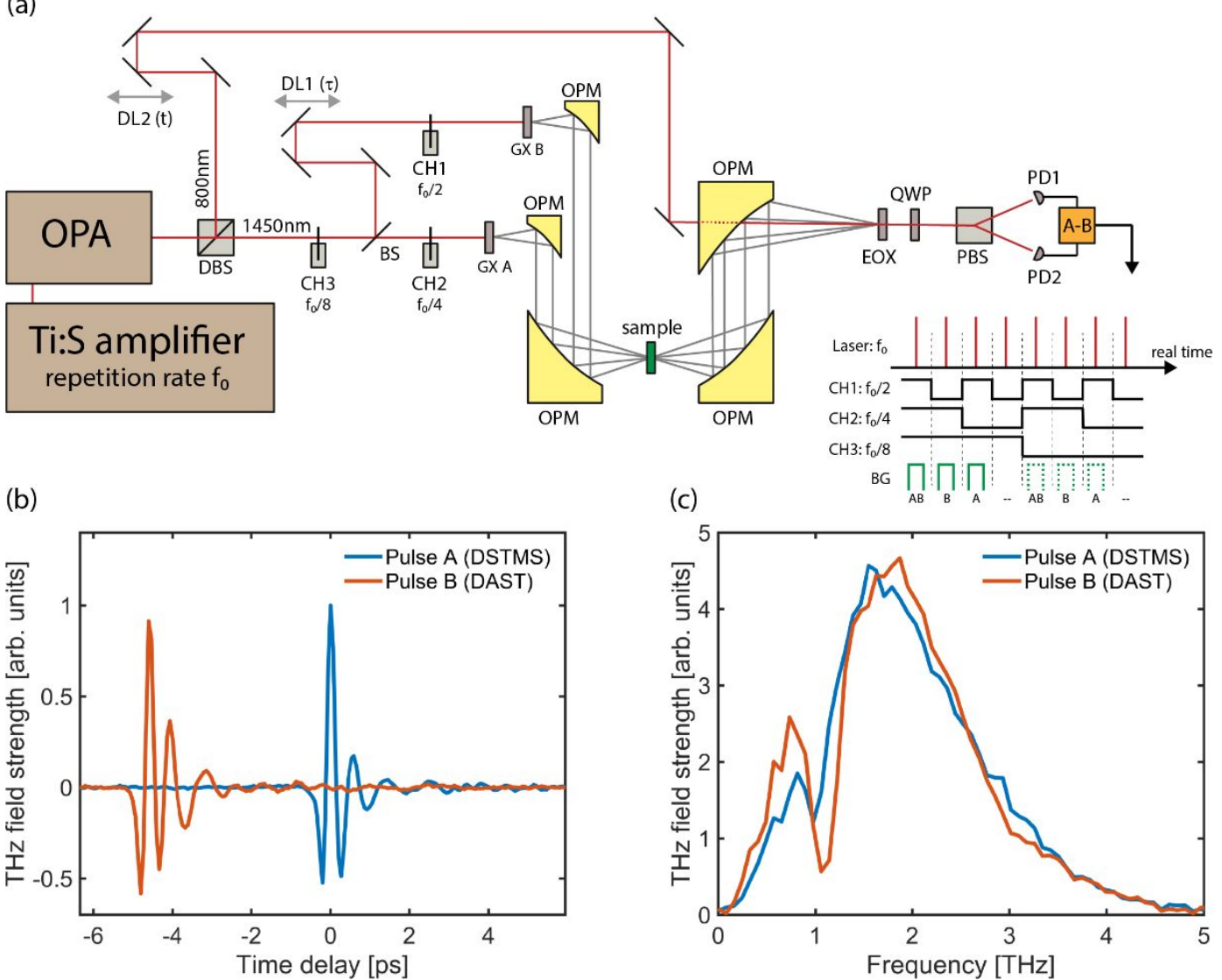

Figure 2. (a) Schematic diagram of experimental setup for nonlinear 2D THz spectroscopy. Two $\mathrm{THz}$ pulses are generated in independent crystals (GX A and GX B), focused and overlapped at the sample position, and guided to electro-optic detection. A chopper scheme allows simultaneous recording pulse A only, pulse B only, and pulse A and B together. Pulse A and B are separated by a variable delay $\tau$. (b) Representative time traces of pulse A and pulse B. (c) Frequency spectra of the two pulses.

\section{Nonlinear interactions in doped $\mathrm{SiC}$}

At least three mechanisms contribute to the observed nonlinearities. However, we find that one of these mechanisms (field-driven electron tunnel ionization of dopants) completely dominates the observed nonlinearities.

Strong $\mathrm{THz}$ fields can induce dopant state ionization. The $\mathrm{THz}$ electric field can lower the potential barrier of a bound dopant electron sufficiently to open a tunneling channel to the conduction band, and even lead to above-barrier field ionization of the dopant states in sufficiently strong fields. For a hydrogen-like donor state with estimated binding energy of $E_{D}=m_{e}^{*} E_{H} /\left(m_{0} \varepsilon_{r}^{2}\right)=61 \mathrm{meV}$ and Bohr radius of $a_{D}=\varepsilon_{r} \varepsilon_{0} h^{2} /\left(m_{e}^{*} e^{2} \pi\right)=1.2 \mathrm{~nm}$, the critical field for above-barrier ionization is $F_{T H z}=E_{D} /\left(e a_{D}\right)=0.5 \mathrm{MV} / \mathrm{cm}[51,52]$. This shows that the $\mathrm{THz}$ electric field accessible in our experiments is sufficient to induce significant tunneling 
release dopant state electrons into the conduction band, and possibly reach into the abovebarrier ionization regime. The dopant Bohr radius $(1.2 \mathrm{~nm})$ is smaller than the average distance between the dopant atoms of $6.3 \mathrm{~nm}$ for the dopant concentration $N_{D}=3.95 \cdot 10^{18} \mathrm{~cm}^{-3}$. Thus, the dopants are not expected to form a miniband below the conduction band, and only carriers excited to the conduction band contribute to the $\mathrm{THz}$ absorption.

Other mechanisms can contribute to the nonlinearities of carriers in SiC. Firstly, the strong $\mathrm{THz}$ field can accelerate electrons to kinetic energies above the energetic difference between the two lowest conduction band minima $(0.12 \mathrm{eV}$ in $4 H$-SiC $[14,16]$, see Figure $1(\mathrm{e}))$, so that electrons can scatter to the second conduction band. Assuming ballistic acceleration in a $100 \mathrm{kV} / \mathrm{cm}$ field, a conduction band electron reaches $0.15 \mathrm{eV}$ kinetic energy within less than $100 \mathrm{fs}$. The transverse mass of electrons in the second conduction band is slightly smaller than that of electrons in the first conduction band $[16,53]$, corresponding to a higher mobility $\mu=e \tau / \mathrm{m}^{*}$ in the second conduction band. Thus, inter-conduction band scattering can lead to a nonlinear strong-field response. Secondly, the conduction band structure of $4 \mathrm{H}-\mathrm{SiC}$ is anharmonic near the $\mathrm{M}$ point, leading to an energy-dependent effective mass $\left(m^{*}(\varepsilon)=m_{0}^{*}(1+\alpha \varepsilon)\right.$ where $\varepsilon$ is the kinetic energy of the electrons), leading to a reduced mobility when carrier are strongly driven in the conduction band. These two effects thus counteract each other, minimizing their detectable influence on the nonlinear response. By using known literature values for the effective masses [16] and anharmonicity factor $\alpha[16,54]$, we have confirmed that these effects have insignificant impact on the nonlinearity compared to the tunnel ionization dynamics.

\section{Modelling of the nonlinear dynamics}

We have implemented a simple rate equation model that incorporates dopant state ionization by THz-induced tunneling. The total density of thermally excited free carriers before the arrival of the $\mathrm{THz}$ pulses is determined as a function of temperature from the neutrality equation considering only nitrogen dopant which is dominant contributor of free carriers [55],

$$
\begin{aligned}
N_{0}+N_{a}= & \frac{N_{d h}}{1+} g_{h} \exp \left(E_{d h} / k_{B} T\right)+g_{v o h} \exp \left(\left(E_{d h}-\Delta E_{v o h}\right) / k_{B} T\right) \\
& +\frac{N_{d k}}{1+g_{k} \exp \left(E_{d k} / k_{B} T\right)+g_{v o k} \exp \left(\left(E_{d k}-\Delta E_{v o k}\right) / k_{B} T\right)} .
\end{aligned}
$$

We use dopant state concentrations $N_{d h}=N_{d k}=N_{D} / 2=1.95 \cdot 10^{18} \mathrm{~cm}^{-3}$, given by the $\mathrm{N}$ concentration determined by ToF-SIMS, dopant state ionization energies of $E_{d h}=41 \mathrm{meV}$ and $E_{d k}=89 \mathrm{meV}$, valley-orbit splitting energies $\Delta E_{v o h}=7.1 \mathrm{meV}$ and $\Delta E_{v o k}=45.5 \mathrm{meV}$, and 
degeneracy parameters $g_{h}=g_{k}=2$ and $g_{v o h}=g_{v o h}=4$ [55]. With these literature values, we reproduce the room-temperature, field-free carrier concentration in the conduction band $N_{0}=6.1 \cdot 10^{16} \mathrm{~cm}^{-3}$ as inferred from our THz-TDS measurements. In spite of the high acceptor dopant concentration, the high density of free electrons indicates that a significant portion of the $\mathrm{B}$ dopants are not electrically active to effectively compensate the $\mathrm{N}$ dopants, and that the sample is effectively uncompensated $\left(N_{A} \approx 0\right)$.

The tunneling and impact ionization rates of dopant states of $4 H-\mathrm{SiC}$ are unknown and for the sake of simplicity, we consider tunneling as the only ionization mechanism. The tunneling rate $\gamma_{Z}$ of electrons from the dopant state to the conduction band can be described as $[52,56,57]$

$$
\gamma_{Z}=\beta\left(\frac{6 \alpha}{F_{T H z}}\right)^{2 n^{*}-1} \exp \left(-\frac{\alpha}{F_{T H z}}\right),
$$

where $\alpha, \beta$ and $n^{*}$ are tunneling parameters that depend on the ionization energy of the dopants. Since no literature values for these parameters exist for $\mathrm{N}$ dopants in SiC, except idealized theoretical expressions, we treat them as fitting parameters.

Using the field-dependent ionization rate, the kinetics of the carrier concentration $N(t)$ in the lowest conduction band is

$$
\frac{d N}{d t}=\gamma_{Z} N_{D}-\gamma_{r e c} \Delta N
$$

Here $N_{D}$ is the carrier concentration in the dopant states, $\Delta N=N(t)-N_{0}$ is the excess carrier concentration in the conduction band due to thermal excitation and tunnel ionization, and $\gamma_{\text {rec }}$ is a phenomenological carrier recombination rate from conduction band to dopant state. Equation (5), coupled with the field-dependent ionization rate (Eq. (4)) allows us to calculate the carrier concentration at a given time and position in the sample. We model the optical interaction by the Drude model of electrical conductivity, $\sigma(\omega)=\sigma_{d c} /\left(1-i \omega \tau_{s}\right)$, where the DC conductivity is determined by the instantaneous carrier concentration, scattering time and effective mass by $\sigma_{d c}(t, T)=N(t, T) e^{2} \tau_{s}(T) / m^{*}$.

The sample has a finite thickness, and the THz attenuation is high (14.5\% transmission at room temperature). Thus, the incident THz field is attenuated fast as it propagates through the sample, and the nonlinear response therefore obviously depends on the position in the sample. Therefore, in order to model the measured transmission coefficient of the sample, we perform a one-dimensional numerical propagation of an incident $\mathrm{THz}$ field through the sample. The 
nonlinear part of the permittivity becomes a function of position, and a nonlocal function of time due to the memory of the carrier concentration. Therefore, we use finite-difference timedomain (FDTD) propagation for a realistic modelling of the propagation of a strong THz field through the sample.

We use the frequency-dependent FDTD method useful for dispersive materials [58, 59], formulated with the flux density and using the Z-transform formalism for the description of the permittivity, as described by Sullivan [60]. In this formalism, the propagation of the field is governed by the standard Yee method [61], and the frequency-dependent nonlinear permittivity describes the nonlinearity of the system. The propagation functions and relation between the flux density and the electric fields are

$$
\begin{aligned}
\frac{\partial \mathbf{D}}{\partial t} & =\nabla \times \mathbf{H} \\
\varepsilon_{0} \varepsilon_{r}^{*}(\omega) \mathbf{E}(\omega) & =\mathbf{D}(\omega) \\
\frac{\partial \mathbf{H}}{\partial t} & =\frac{1}{\mu_{0}} \nabla \times \mathbf{E}
\end{aligned}
$$

Where the relative permittivity is determined by the linear properties (Figure 1) and the additional conductivity of the field-induced carrier concentration. In order to move from the frequency-domain representation to a time-domain representation of the flux density, the constituting relation for $\mathbf{D}(\omega)$ is Fourier transformed to the time domain, and $\mathrm{Z}$ matrix formalism is applied to adapt to the discrete grid of the simulation.

\section{Nonlinear single-pulse experimental results and simulations}

The nonlinear $\mathrm{THz}$ transmission of a single pulse was investigated over the $10-350 \mathrm{~K}$ temperature range by placing the sample in a helium-cooled cryostat. Figure 3(a) shows two $\mathrm{THz}$ transients with incident peak field strengths of 33 and $280 \mathrm{kV} / \mathrm{cm}$, respectively, after propagation through the sample at room temperature. Both signals have been normalized to the peak strength of the incident field, and the nonlinearity of the transmission can thus be directly compared. The measurements were performed with $N=6 \mathrm{HR}-\mathrm{Si}$ wafers placed before the sample $(33 \mathrm{kV} / \mathrm{cm})$ or after the sample $(280 \mathrm{kV} / \mathrm{cm})$. In this manner, the detected signals are directly comparable in amplitude. The refractive index of undoped $4 \mathrm{H}$-SiC is approximately 3.12 in the $0-2 \mathrm{THz}$ range [12], yielding a field transmission of 0.735 due to Fresnel reflection losses. The low-field peak $\mathrm{THz}$ transmission of only 0.143 at room temperature is due to freecarrier absorption (FCA) of the significant density of thermally activated free carriers from the $\mathrm{N}$ dopant states. 

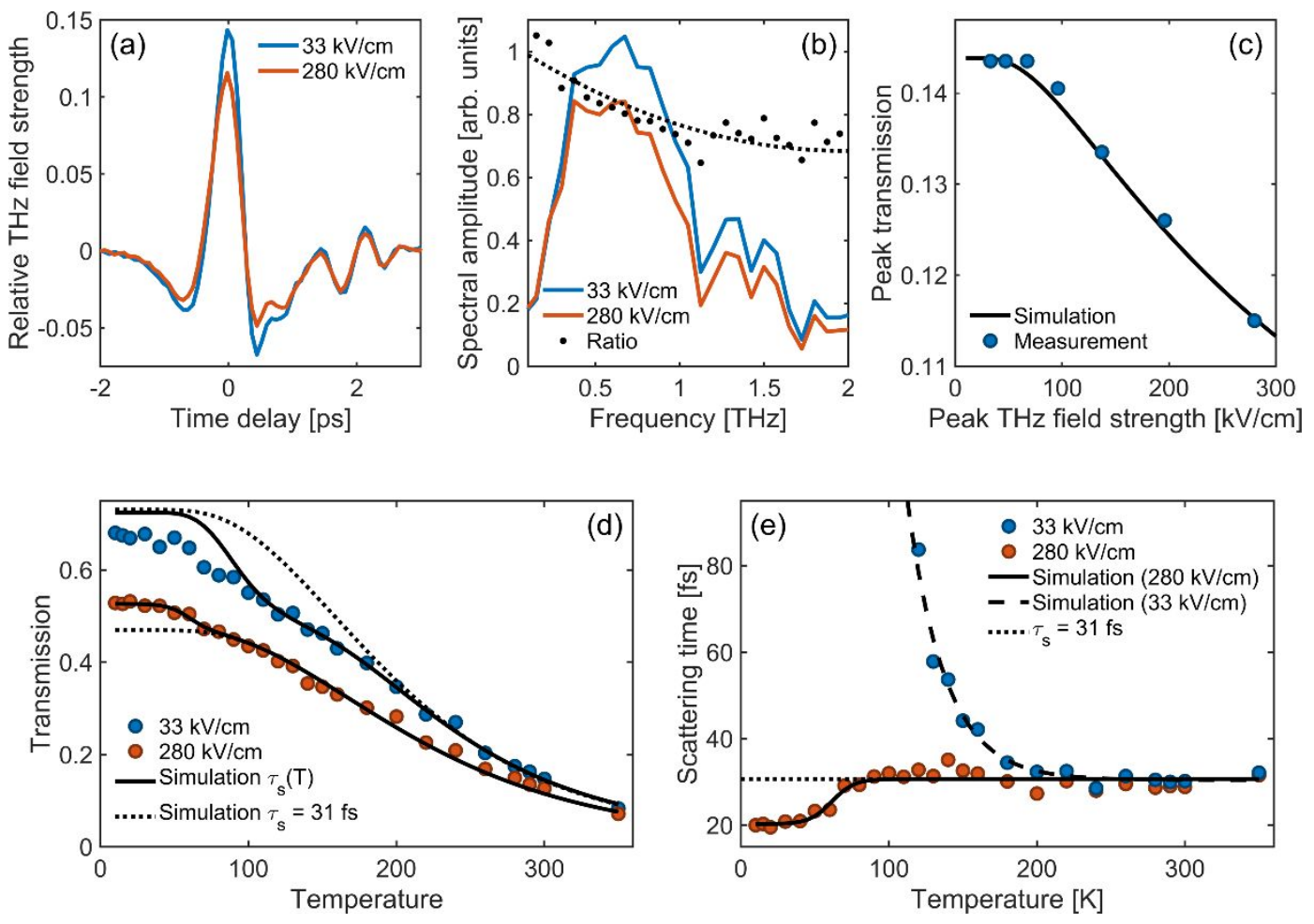

Figure 3. (a) Time traces of transmitted THz signals at 33 and $280 \mathrm{kV} / \mathrm{cm}$ peak field strength. The nonlinear response of $\mathrm{SiC}$ leads to decreased transmission. (b) Corresponding frequency spectra and ratio of the spectra (black data points and averaged, dashed curve). (c) Transmission through the SiC sample as function of peak incident field strength. Blue symbols are measured values, black curve is the nonlinear FDTD simulation result. (d) Temperature-dependent transmission at 33 and 280 $\mathrm{kV} / \mathrm{cm}$ peak field strength, with nonlinear FDTD simulation results using temperature- and fielddependent scattering time (solid curves) and constant scattering time (dashed curves). (e) Temperature-dependent scattering time extracted from nonlinear FDTD simulations.

Figure 3(b) shows the spectra of the two pulses, extending from 0.1 to $2 \mathrm{THz}$. The dips in the spectra are due to the well-known water absorption lines in the low $\mathrm{THz}$ range [62]. The ratio of the two spectra is shown as black symbols and a dashed curve to guide the eye. The nonlinear response reduces the transmission by approximately $15-20 \%$ across the $0.1-2 \mathrm{THz}$ range. The blue symbols in Figure 3(c) shows the peak value of the THz field after the sample relative to the peak input field strength (the peak transmission) as function of the incident field strength. The solid black curve is the result of the nonlinear FDTD simulations with values of the Zener tunneling parameters $\alpha=80 \mathrm{kV} / \mathrm{cm}, \beta=9 \cdot 10^{10} \mathrm{~s}^{-1}$ and $n=0.6$. We used a room-temperature scattering time of $30.7 \mathrm{fs}$, within the estimated uncertainty of the 34 fs obtained in our broadband $\mathrm{THz}$ measurements. We observe an onset of the nonlinearity at $50-70 \mathrm{kV} / \mathrm{cm}$, followed by an approximately linear decrease in transmission up to the highest field strength available in the experiment. The agreement between simulation and experiment indicates that the nonlinear response can be attributed to field-driven ionization of the $\mathrm{N}$ dopant states. The 
large bandgap energy of SiC inhibits $\mathrm{THz}$-induced free carrier generation by interband Zener tunneling at sub-MV/cm fields.

The temperature dependence of the peak field transmission at 33 and $280 \mathrm{kV} / \mathrm{cm}$ is shown as blue and red symbols, respectively, in Figure 3(d). Together with the experimental results we show two sets of simulated transmission curves. In all simulations, the temperature-dependent initial carrier concentration is determined by the neutrality equation (Eq. (3)). The dashed curves are obtained with a scattering time independent of temperature (30.7 fs), and the solid curves show the simulated transmission using temperature-dependent scattering times.

The temperature-dependent scattering time was optimized by minimizing the root-mean-square (RMS) distance between the experimental and simulated transmission at low and high fields, respectively. The resulting temperature-dependent scattering times are shown in Figure 3(e). For the low-field transmission, we find an apparent divergence of the scattering time for temperatures below $180 \mathrm{~K}$. At strong fields we do not observe this divergence of the scattering time. The best-fit scattering time remains constant from room temperature to $80 \mathrm{~K}$, and even reduces gradually to 20 fs towards the lowest temperature of $10 \mathrm{~K}$ in the measurements.

The temperature dependence of the low-field scattering time can be understood by considering the temperature dependence of the various scattering mechanisms that contribute to the total scattering time. We find qualitatively similar temperature dependence of the scattering time as observed in Hall mobility measurements [27, 55, 63]. Following the interpretation and calculations by Pernot et al. [55], the electron scattering time in $n$-type $4 H$-SiC is dominated by intervalley phonon scattering at room temperature, whereas intravalley acoustic scattering and ionized/neutral impurity scattering start to contribute to the total scattering rate at intermediate temperatures. At the lowest temperatures, most scattering mechanisms are suppressed due to freeze-out of phonons and insignificant thermal ionization of dopants, so neutral impurity scattering will dominate. The agreement between the low-field simulation and experimental observations is not perfect at temperatures below $100 \mathrm{~K}$. The carrier concentration below 100 $\mathrm{K}$ and at low field strength is so low that the transmission is close to that of an undoped sample, and the predictive power of the simulation is much lower than at room temperature.

In the strong-field case, the constant scattering time of $30 \mathrm{fs}$ between 80 and $300 \mathrm{~K}$ suggests that intervalley phonon scattering remains active. Also, ionized impurity scattering remains important at lower temperatures due to the field-driven ionization of the dopants. The drop in the total scattering time below $80 \mathrm{~K}$ can be related to the decreased ionized impurity scattering time at the lowest temperatures that contributed to a similar drop in Hall mobility in [55]. 


\section{Nonlinear two-dimensional spectroscopy of the carrier dynamics}

Single-pulse transmission (Figure 3(a)) shows that the nonlinear effect reduces the transmission of the pulse itself, and thus the involved time constants must be much shorter than the duration of the $\mathrm{THz}$ pulse. It is therefore interesting to investigate the temporal dynamics of the nonlinearity. To this end, we have performed nonlinear two-dimensional THz spectroscopy of the sample.

Figure 4 shows an overview of the nonlinear 2D THz experimental data recorded at full field strength. The top panels (Figure 4(a)-(c)) show the raw signals from the experiments, with both THz pulses present $\left(E_{A B}\right)$, and with pulse $\mathrm{A}$ and pulse B alone ( $E_{A}$ and $E_{B}$, respectively). The signals have been scaled by a common factor so that the peak field strength of pulse A is unity. The horizontal axis represents the time trace of the THz signals, as shown in Figure 2(b), and the color scale represents the positive (red) and negative (blue) field strength. The vertical axis represents the delay time $\tau$ between the two pulses. Figure 4(d) is the main result, and shows the nonlinear signal $E_{N L}$ (Eq. (2)). The nonlinear 2D signal displays an apparently instantaneous (within the time resolution of the measurement) response during the temporal overlap of pulse A and B, and the nonlinear interference decays rapidly (within $1 \mathrm{ps}$ ) when the two pulses separate in time. This is seen as the signal tails in directions vertically towards negative delay times (pulse B affects the transmission of pulse A) and along the diagonal towards positive $\tau$ and $t$ (pulse A affects the transmission of pulse $\mathrm{B}$ ). This observation is consistent with the single-pulse observation of the nonlinearity acting on the pulse itself, with sub-cycle dynamics on a time scale much shorter than the duration of the pulse. 

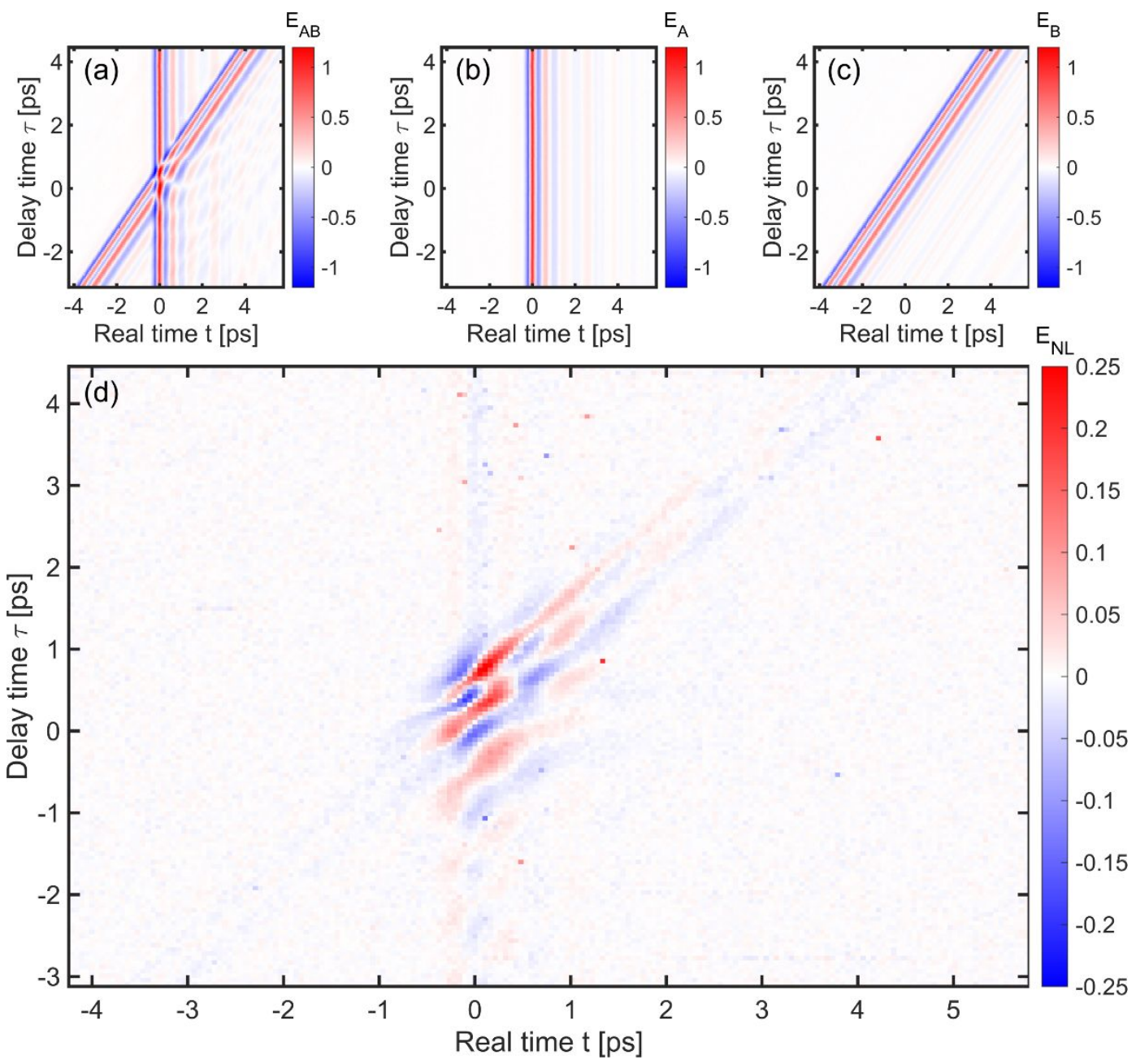

Figure 4. Time-domain representation of nonlinear two-dimensional $\mathrm{THz}$ spectroscopy of the $\mathrm{SiC}$ sample. (a) Two THz pulses (A and B) interfering in the sample as function of the delay time between A and B, (b) pulse A alone, (c) pulse B alone, and (d) the nonlinear response, defined as $E_{N L}(t, \tau)=E_{A B}(t, \tau)-E_{A}(t, \tau)-E_{B}(t, \tau)$.

The nonlinear signal reaches an amplitude of $25 \%$ of the pump signals, indicative of a very strong nonlinearity. However, we note that such large amplitude of the nonlinearity can be caused by both nonlinear absorption and nonlinear phase shift of the signals. The nonlinear signal is extremely sensitive to drifts of the $\mathrm{AB}, \mathrm{A}$ and $\mathrm{B}$ signals during the long measurements. A full 2D measurement as shown in Figure 4 takes approximately 12 hours, so any drift in power or pointing of the laser system will influence the measurements and potentially lead to erroneous apparent nonlinearities after the signal subtraction. To ensure that the measured signals originate from the nonlinearity of the sample, we performed the measurement several times at full field strength, with identical results. Additional reference measurements without the sample in the beam path where nonlinearities are not expected at our signal strengths were also carried out, and we confirmed that we did not detect a nonlinear signal in that case, 
confirming the linearity of our electro-optic detection. Furthermore, we performed the measurements at three different field strengths $\left(E_{\max }, E_{\max } / 2, E_{\max } / 4\right)$.

Figure 5(a) shows the 2D Fourier transform of the nonlinear time-domain signal shown in Figure 4(d). The horizontal axis, labeled $v_{t}$, represents the probe frequency, and the vertical axis, labeled $v_{\tau}$, represents the pump frequency. Due to the arrangement of the time traces, the spectral signal of pulse A is distributed along the horizontal axis, and the spectral signal of pulse $\mathrm{B}$ is distributed along the negative diagonal $\left(v_{t}=-v_{\tau}\right)$ of the $2 \mathrm{D}$ spectrum. The dashed lines in Figure 5(a) indicate the location of the most important contributions to the nonlinear signal. Following the method and nomenclature of Kuehn et al. [49] the lines labeled $k_{A}$ and $k_{B}$ are the pump-probe signals, where pulse A is modulated by pulse B and pulse B is modulated by pulse A, respectively. The line labeled $2 k_{B}-k_{A}$ indicate the expected position of the third-order four-wave mixing signal, or two-photon echo. The line labeled $k_{A}+k_{B}$ is the expected position of a sum-frequency generation (SFG) signal. Figure 5(b) shows the amplitude of the nonlinear signal along these directions, plotted as functions of the probe frequency $v_{t}$. The inset of Figure 5(b) shows the field dependence of the integrated strength of the nonlinear signals. The symbols represent the experimental data, and the dashed curves are linear fits to the PP signals and quadratic fits to the FWM and SFG signals. We determined that these polynomial orders gave the best fits to the few data points. The linear field dependence of the nonlinear pump-probe signals is consistent with the approximately linear dependence of the peak transmission on the field strength (Figure 3(c)). The quadratic field dependence of the second-order nonlinearity (SFG) is as expected, whereas the third-order FWM nonlinearity should in principle show a cubic dependence on field strength. The apparent lower-order field dependence of the FWM signal can be due to saturation or onset of nonperturbative response of the sample, or could be affected by the nearby stronger pump-probe signal, as we see a significant overlap of the two parts of the signal in the $2 \mathrm{D}$ spectrum.

The appearance of distinct higher-order nonlinearities in the 2D spectrum is a manifestation of the specific field-dependence of the nonlinearity that can be expanded to higher orders with decreasing magnitude in the perturbative picture. Since the interaction is largely nonresonant (the Drude absorption spectrum of the free carriers is broad and featureless), the nonlinear signals are, likewise, broad spectra that resemble the pump spectrum. We note that undoped $4 \mathrm{H}$-SiC is an efficient second-order medium for optically pumped $\mathrm{THz}$ generation [64] and second-harmonic generation in the mid-infrared [65], so in principle, the observed SFG signal 
can have a contribution both from the carrier-induced nonlinearity and from the second-order nonlinearity of the lattice. At this point, however, we are not able to separate these two contributions.

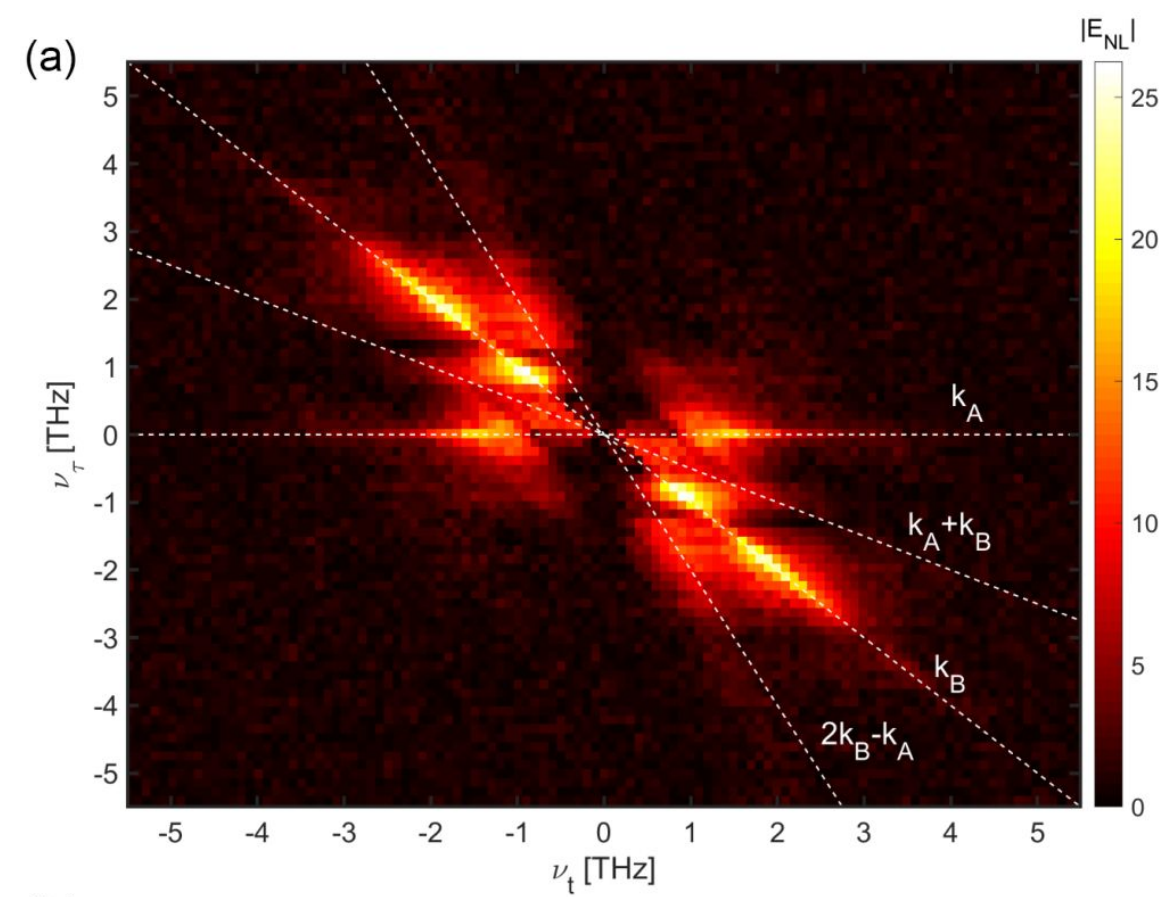

(b)

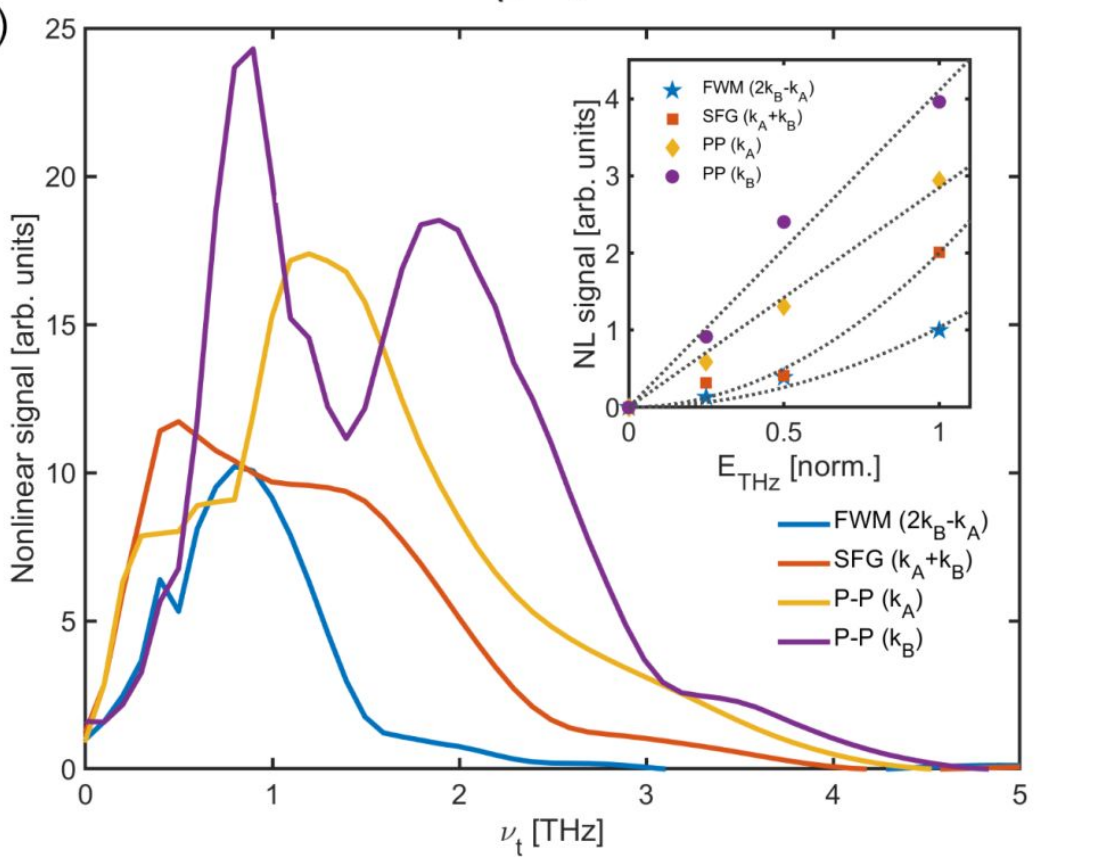

Figure 5. (a) Nonlinear 2D frequency spectrum of the nonlinear time-domain data shown in Figure 4, defined as $\left|E_{N L}\left(v_{t}, v_{\tau}\right)\right|=\left|E_{A B}\left(v_{t}, v_{\tau}\right)-E_{A}\left(v_{t}, v_{\tau}\right)-E_{B}\left(v_{t}, v_{\tau}\right)\right|$. Dashed lines indicate the expected positions of the pump-probe signals $\left(k_{A}\right.$ and $\left.k_{B}\right)$, sum frequency generation $\left(k_{A}+k_{B}\right)$, and fourwave mixing $\left(2 k_{B}-k_{A}\right)$. (b) Line plots of the nonlinear signal along the indicated directions in (a). The inset shows the field dependence of the nonlinear signal contributions. Dashed curves are linear (PP) and quadratic (FWM, SFG) fits. 
The detected spectral shape of the nonlinear signals is a result of the nonlinear interaction between the fields as well as the propagation of the fields during and after the nonlinear interaction. Due to the slight angle between the pump beams, the nonlinear signals are emitted into frequency-dependent angles and thus the detected spectral shape is influenced by the numerical aperture of the collecting off-axis paraboloidal mirror, hindering a precise analysis of the spectral shapes. However, we observe that the FWM signal in Figure 5(b) (blue curve) is centered at $1 \mathrm{THz}$ and extends to approximately $2 \mathrm{THz}$, whereas the SFG signal (red curve) has a broader spectral content reaching to above $3 \mathrm{THz}$. The pump-probe signals have spectral shapes similar to the spectra of the respective pump pulses (see Figure 2(c)).

\section{Conclusions}

In conclusion, we show that $\mathrm{SiC}$, which is one of the most radiation resistant materials, can be tailored to have extremely fast $\mathrm{THz}-$ induced nonlinear behavior in moderate $\mathrm{THz}$ fields by addition of appropriate dopants. This is demonstrated by investigating doped $4 \mathrm{H}$-SiC sample which contains high concentrations of nitrogen and boron dopants. As the THz field strength increases, transmission decreases approximately linearly for electric fields higher than approximately $60 \mathrm{kV} / \mathrm{cm}$. We describe the nonlinearity by a simple model of the carrier kinetics in the conduction band, where carriers driven by the strong $\mathrm{THz}$ field can tunnel from the $\mathrm{N}$ dopant sites to the conduction band. Nonlinear two-dimensional THz spectroscopy revealed that the nonlinearity consists of incoherent pump-probe signals, second-order signals consistent with sum-frequency generation, and third-order four-wave mixing signals.

The ultrafast THz-induced increase in absorption that we show here may be useful in technological applications that require transmission bleaching, such as signal limiters in optical signal processing. While extremely fast modulation of transmission can be achieved by free carrier generation through ultrafast impact ionization and tunneling processes in other semiconductors $[11,66,67]$, the recovery time of these nonlinear modulation processes are comparably slow. Here we have shown that $\mathrm{SiC}$ can be used to make extremely fast modulation with full recovery within approximately one picosecond, a feature that could become applicable in ultrafast all-optical nonlinear signal processing at $\mathrm{THz}$ frequencies, especially if combined with field enhancement techniques to overcome the requirement of the very high field strengths used here. 


\section{Funding acknowledgments}

K.J.K. and P.U.J. gratefully acknowledge financial support by the Center for Nanostructured Graphene (CNG), which is sponsored by the Danish National Research Foundation, Project No. DNRF103. P.U.J. acknowledges partial financial support from the Danish Innovation Fund (Project TRIM).

\section{References}

[1] M. B. J. Wijesundara, and R. Azevedo, Silicon Carbide Microsystems for Harsh Environments (Springer, 2011).

[2] J. B. Casady, and R. W. Johnson, "Status of silicon carbide ( $\mathrm{SiC}$ ) as a wide-bandgap semiconductor for hightemperature applications: A review," Solid-State Electronics 39, 1409-1422 (1996).

[3] M. R. Werner, and W. R. Fahrner, "Review on materials, microsensors, systems and devices for hightemperature and harsh-environment applications," IEEE Transactions on Industrial Electronics 48, 249-257 (2001).

[4] H. Tamaso, K. Sawada, K. Fujikawa, S. Harada, J. Shinkai, H. Tokuda, T. Masuda, M. Honaga, S. Itoh, T. Tsuno, and Y. Namikawa, "Development of Fast-Switching SiC Transistor," SEI Technical Review 66, 43-49 (2008).

[5] J. H. Zhao, V. Gruzinskis, Y. Luo, M. Weiner, M. Pan, P. Shiktorov, and E. Starikov, "Monte Carlo simulation of 4H-SiC IMPATT diodes," Semicond. Sci. Tech. 15, 1093 (2000).

[6] J. Dai, J. Zhang, W. Zhang, and D. Grischkowsky, "Terahertz time-domain spectroscopy characterization of the far-infrared absorption and index of refraction of high-resistivity, float-zone silicon," J. Opt. Soc. Am. B 21, 1379-1386 (2004).

[7] H. Hirori, A. Doi, F. Blanchard, and K. Tanaka, "Single-cycle terahertz pulses with amplitudes exceeding 1 $\mathrm{MV} / \mathrm{cm}$ generated by optical rectification in $\mathrm{LiNbO}_{3}$," Appl. Phys. Lett. 98, 091106 (2011).

[8] K. Iwaszczuk, A. Andryieuski, A. Lavrinenko, X. C. Zhang, and P. U. Jepsen, "Terahertz field enhancement to the MV/cm regime in a tapered parallel plate waveguide," Opt. Express 20, 8344-8355 (2012).

[9] C. P. Hauri, C. Ruchert, C. Vicario, and F. Ardana, "Strong-field single-cycle THz pulses generated in an organic crystal," Appl. Phys. Lett. 99, 161116 (2011).

[10] C. Vicario, B. Monoszlai, and C. P. Hauri, "GV/m single-cycle terahertz fields from a laser-driven large-size partitioned organic crystal," Phys. Rev. Lett. 112, 213901 (2014).

[11] A. Tarekegne, T. , K. Iwaszczuk, M. Zalkovskij, A. C. Strikwerda, and P. U. Jepsen, "Impact ionization in high resistivity silicon induced by an intense terahertz field enhanced by an antenna array," New J. Phys. 17, $043002(2015)$.

[12] A. T. Tarekegne, B. Zhou, K. Kaltenecker, K. Iwaszczuk, S. Clark, and P. U. Jepsen, "Terahertz time-domain spectroscopy of zone-folded acoustic phonons in 4H and 6H silicon carbide," Opt. Express 27, 3618-3628 (2019). [13] P. Käckell, B. Wenzien, and F. Bechstedt, "Electronic properties of cubic and hexagonal SiC polytypes from ab initio calculations," Phys. Rev. B 50, 10761-10768 (1994).

[14] B. Kaczer, H. J. Im, J. P. Pelz, J. Chen, and W. J. Choyke, "Direct observation of conduction-band structure of 4H- and 6H-SiC using ballistic electron emission microscopy," Phys. Rev. B 57, 4027-4032 (1998).

[15] B. Wenzien, P. Käckell, F. Bechstedt, and G. Cappellini, "Quasiparticle band structure of silicon carbide polytypes," Phys. Rev. B 52, 10897-10905 (1995).

[16] C. Persson, and U. Lindefelt, "Relativistic band structure calculation of cubic and hexagonal SiC polytypes," J. Appl. Phys. 82, 5496-5508 (1997).

[17] G. Pennington, and N. Goldsman, "Self-consistent calculations for n-type hexagonal SiC inversion layers," J. Appl. Phys. 95, 4223-4234 (2004).

[18] G. Sharma, I. Al-Naib, H. Hafez, R. Morandotti, D. G. Cooke, and T. Ozaki, "Carrier density dependence of the nonlinear absorption of intense THz radiation in GaAs," Opt. Express 20, 18016-18024 (2012).

[19] T. Terashige, H. Yada, Y. Matsui, T. Miyamoto, N. Kida, and H. Okamoto, "Temperature and carrier-density dependence of electron-hole scattering in silicon investigated by optical-pump terahertz-probe spectroscopy," Phys. Rev. B 91, 241201 (2015).

[20] S. Li, G. Kumar, and T. E. Murphy, "Terahertz nonlinear conduction and absorption saturation in silicon waveguides," Optica 2, 553-557 (2015).

[21] J. Hebling, M. C. Hoffmann, H. Y. Hwang, K. L. Yeh, and K. A. Nelson, "Observation of nonequilibrium carrier distribution in $\mathrm{Ge}, \mathrm{Si}$, and GaAs by terahertz pump-terahertz probe measurements," Phys. Rev. B 81, $035201(2010)$. 
[22] D. Turchinovich, J. M. Hvam, and M. C. Hoffmann, "Self-phase modulation of a single-cycle terahertz pulse by nonlinear free-carrier response in a semiconductor," Phys. Rev. B 85, 201304 (2012).

[23] F. Blanchard, D. Golde, F. H. Su, L. Razzari, G. Sharma, R. Morandotti, T. Ozaki, M. Reid, M. Kira, S. W. Koch, and F. A. Hegmann, "Effective Mass Anisotropy of Hot Electrons in Nonparabolic Conduction Bands of nDoped InGaAs Films Using Ultrafast Terahertz Pump-Probe Techniques," Phys. Rev. Lett. 107, 107401 (2011).

[24] C. H. Park, B.-H. Cheong, K.-H. Lee, and K. J. Chang, "Structural and electronic properties of cubic, 2H, 4H, and 6H SiC," Phys. Rev. B 49, 4485-4493 (1994).

[25] N. T. Son, W. M. Chen, O. Kordina, A. O. Konstantinov, B. Monemar, E. Janzén, D. M. Hofman, D. Volm, M. Drechsler, and B. K. Meyer, "Electron effective masses in 4H SiC," Appl. Phys. Lett. 66, 1074-1076 (1995).

[26] X. Liu, S.-Y. Zhuo, P. Gao, W. Huang, C.-F. Yan, and E.-W. Shi, "Donor-acceptor-pair emission in fluorescent 4H-SiC grown by PVT method," AIP Advances 5, 047133 (2015).

[27] W. Götz, A. Schöner, G. Pensl, W. Suttrop, W. J. Choyke, R. Stein, and S. Leibenzeder, "Nitrogen donors in 4H-silicon carbide," J. Appl. Phys. 73, 3332-3338 (1993).

[28] T. Kimoto, and J. A. Cooper, Fundamentals of Silicon Carbide Technology (John Wiley \& Sons Singapore Pte. Ltd, 2014).

[29] M. Ikeda, H. Matsunami, and T. Tanaka, "Site effect on the impurity levels in 4H, 6H, and 15R SiC," Phys. Rev. B 22, 2842-2854 (1980).

[30] R. Mickevičius, and J. H. Zhao, "Monte Carlo study of electron transport in SiC," J. Appl. Phys. 83, 31613167 (1998).

[31] T. Troffer, M. Schadt, T. Frank, H. Itoh, G. Pensl, J. Heindl, H. P. Strunk, and M. Maier, "Doping of SiC by implantation of boron and aluminum," Phys. Status Solidi A 162, 277-298 (1997).

[32] J. Pernot, S. Contreras, and J. Camassel, "Electrical transport properties of aluminum-implanted 4H-SiC," J. Appl. Phys. 98, 023706 (2005).

[33] A. A. Lebedev, "Deep level centers in silicon carbide: A review," Semiconductors 33, 107-130 (1999).

[34] J. W. Sun, S. Kamiyama, V. Jokubavicius, H. Peyre, R. Yakimova, S. Juillaguet, and M. Syväjärvi, "Fluorescent silicon carbide as an ultraviolet-to-visible light converter by control of donor to acceptor recombinations," J. Phys. D 45, 235107 (2012).

[35] D. J. Cook, and R. M. Hochstrasser, "Intense terahertz pulses by four-wave rectification in air," Opt. Lett. 25, 1210-1212 (2000).

[36] M. Kress, T. Löffler, S. Eden, M. Thomson, and H. G. Roskos, "Terahertz-pulse generation by photoionization of air with laser pulses composed of both fundamental and second-harmonic waves," Opt. Lett. 29, 1120-1122 (2004).

[37] H. G. Roskos, M. D. Thomson, M. Kreß, and T. Löffler, "Broadband THz emission from gas plasmas induced by femtosecond optical pulses: From fundamentals to applications," Laser Photon. Rev. 1, 349-368 (2007).

[38] X. Xie, J. M. Dai, and X. C. Zhang, "Coherent control of THz wave generation in ambient air," Phys. Rev. Lett. 96, 075005 (2006).

[39] J. Dai, X. Xie, and X. C. Zhang, "Detection of broadband terahertz waves with a laser-induced plasma in gases," Phys. Rev. Lett. 97, 103903 (2006).

[40] T. Wang, K. Iwaszczuk, E. A. Wrisberg, E. V. Denning, and P. U. Jepsen, "Linearity of Air-Biased Coherent Detection for Terahertz Time-Domain Spectroscopy," J. Infrared Millim. Terahertz Waves 37, 592-604 (2016).

[41] P. U. Jepsen, and B. M. Fischer, "Dynamic range in terahertz time-domain transmission and reflection spectroscopy," Opt. Lett. 30, 29-31 (2005).

[42] P. U. Jepsen, D. G. Cooke, and M. Koch, "Terahertz spectroscopy and imaging - Modern techniques and applications," Laser Photon. Rev. 5, 124-166 (2011).

[43] J. Hebling, K.-L. Yeh, M. C. Hoffmann, B. Bartal, and K. A. Nelson, "Generation of high-power terahertz pulses by tilted-pulse-front excitation and their application possibilities," J. Opt. Soc. Am. B 25, B6-B19 (2008). [44] Q. Wu, and X. C. Zhang, "Free-space electro-optic sampling of terahertz beams," Appl. Phys. Lett. 67, 35233525 (1995).

[45] A. Nahata, D. H. Auston, T. F. Heinz, and C. J. Wu, "Coherent detection of freely propagating terahertz radiation by electro-optic sampling," Appl. Phys. Lett. 68, 150-152 (1996).

[46] P. U. Jepsen, C. Winnewisser, M. Schall, V. Schyja, S. R. Keiding, and H. Helm, "Detection of THz pulses by phase retardation in lithium tantalate," Phys. Rev. E 53, R3052-R3054 (1996).

[47] K. J. Kaltenecker, E. J. R. Kelleher, B. Zhou, and P. U. Jepsen, "Attenuation of THz Beams: A "How to" Tutorial," J. Infrared Millim. Terahertz Waves 40, 878-904 (2019).

[48] A. T. Tarekegne, H. Hirori, K. Tanaka, K. Iwaszczuk, and P. U. Jepsen, "Impact ionization dynamics in silicon by MV/cm THz fields," New J. Phys. 19, 123018 (2017).

[49] W. Kuehn, K. Reimann, M. Woerner, and T. Elsaesser, "Phase-resolved two-dimensional spectroscopy based on collinear n-wave mixing in the ultrafast time domain," J. Chem. Phys. 130, 164503 (2009).

[50] P. Klarskov, Intense Terahertz Sources for 2D Spectroscopy (Ph.D. Thesis, Technical University of Denmark, 2015).

[51] P. Y. Yu, and M. Cardona, Fundamentals of Semiconductors (Springer, 2010). 
[52] Y. Mukai, H. Hirori, and K. Tanaka, "Electric field ionization of gallium acceptors in germanium induced by single-cycle terahertz pulses," Phys. Rev. B 87, 201202 (2013).

[53] D. Volm, B. K. Meyer, D. M. Hofmann, W. M. Chen, N. T. Son, C. Persson, U. Lindefelt, O. Kordina, E. Sörman, A. O. Konstantinov, B. Monemar, and E. Janzén, "Determination of the electron effective-mass tensor in 4H SiC," Phys. Rev. B 53, 15409-15412 (1996).

[54] J. H. Zhao, V. Gruzinskis, Y. Luo, M. Weiner, M. Pan, P. Shiktorov, and E. Starikov, "Monte Carlo simulation of 4H-SiC IMPATT diodes," Semicond. Sci. Tech. 15, 1093-1100 (2000).

[55] J. Pernot, W. Zawadzki, S. Contreras, J. L. Robert, E. Neyret, and L. D. Cioccio, "Electrical transport in ntype 4H silicon carbide," J. Appl. Phys. 90, 1869-1878 (2001).

[56] H.-Q. Nie, and D. D. Coon, "Tunneling of holes from acceptor levels in an applied field," Solid-State Electronics 27, 53-58 (1984).

[57] A. Dargys, and S. Zurauskas, "Tunnel ionization of shallow acceptors and donors in GaAs," J. Phys.: Condens. Matter 7, 2133 (1995).

[58] R. Luebbers, F. P. Hunsberger, K. S. Kunz, R. B. Standler, and M. Schneider, "A frequency-dependent finitedifference time-domain formulation for dispersive materials," IEEE Transactions on Electromagnetic Compatibility 32, 222-227 (1990).

[59] R. J. Luebbers, F. Hunsberger, and K. S. Kunz, "A frequency-dependent finite-difference time-domain formulation for transient propagation in plasma," IEEE Trans. Antennas Propag. 39, 29-34 (1991).

[60] D. M. Sullivan, "Frequency-dependent FDTD methods using Z transforms," IEEE Trans. Antennas Propag. 40, 1223-1230 (1992).

[61] K. S. Yee, "Numerical solution of initial boundary value problems involving maxwell's equations in isotropic media," IEEE Trans. Antennas Propag. 14, 302-307 (1966).

[62] M. van Exter, C. Fattinger, and D. Grischkowsky, "Terahertz time-domain spectroscopy of water vapor," Opt. Lett. 14, 1128-1130 (1989).

[63] H. Iwata, K. M. Itoh, and G. Pensl, "Theory of the anisotropy of the electron Hall mobility in n-type 4H- and 6H-SiC," J. Appl. Phys. 88, 1956-1961 (2000).

[64] M. P. Fischer, J. Bühler, G. Fitzky, T. Kurihara, S. Eggert, A. Leitenstorfer, and D. Brida, "Coherent field transients below $15 \mathrm{THz}$ from phase-matched difference frequency generation in 4H-SiC," Opt. Lett. 42, 26872690 (2017).

[65] A. Paarmann, I. Razdolski, S. Gewinner, W. Schöllkopf, and M. Wolf, "Effects of crystal anisotropy on optical phonon resonances in midinfrared second harmonic response of SiC," Phys. Rev. B 94, 134312 (2016).

[66] H. Hirori, K. Shinokita, M. Shirai, S. Tani, Y. Kadoya, and K. Tanaka, "Extraordinary carrier multiplication gated by a picosecond electric field pulse," Nat. Commun. 2, 594 (2011).

[67] C. Lange, T. Maag, M. Hohenleutner, S. Baierl, O. Schubert, E. R. J. Edwards, D. Bougeard, G. Woltersdorf, and R. Huber, "Extremely Nonperturbative Nonlinearities in GaAs Driven by Atomically Strong Terahertz Fields in Gold Metamaterials," Phys. Rev. Lett. 113, 227401 (2014). 

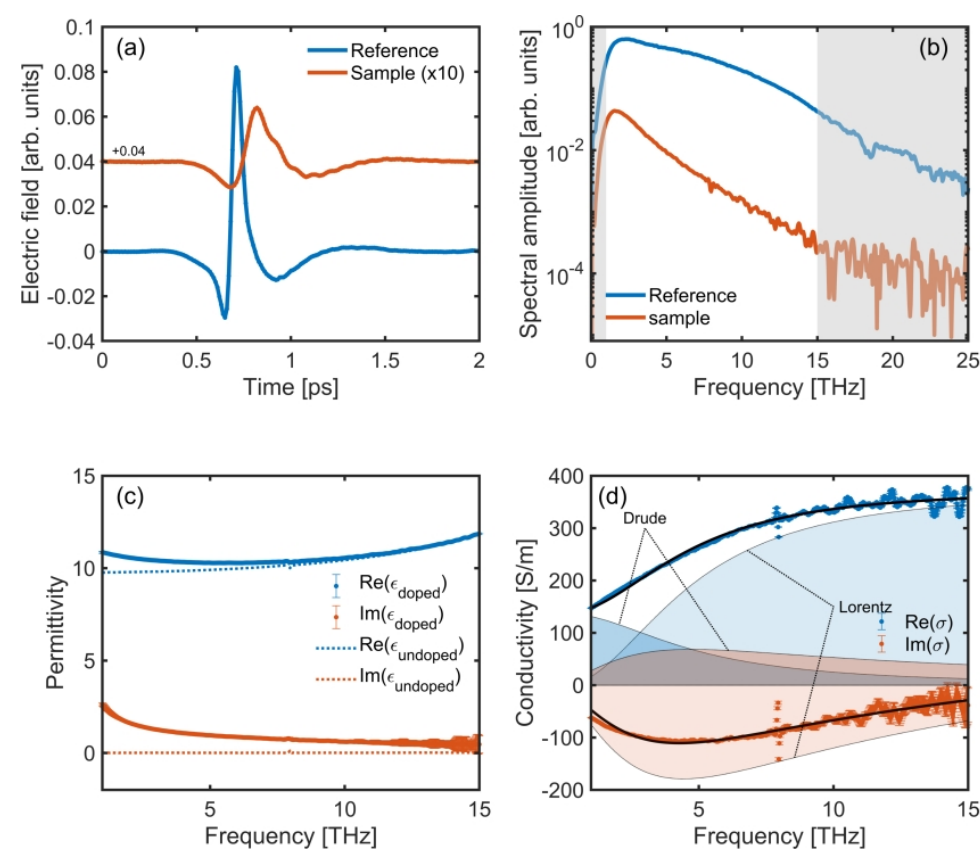

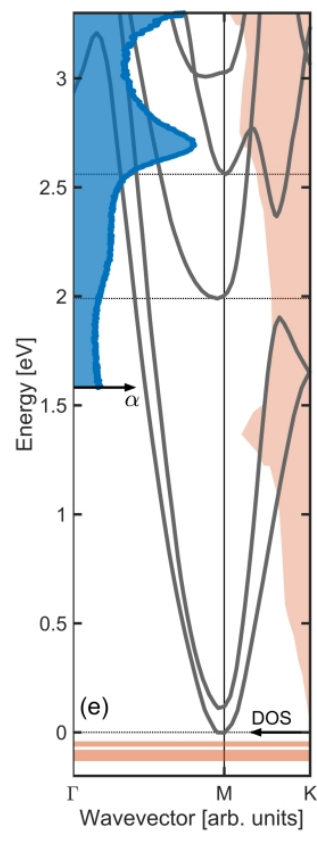

THz-time-domain spectroscopy of co-doped $4 \mathrm{H}$-SiC. (a) Time-domain traces of the reference pulse (blue curve) and sample pulse (red curve). (b) Frequency spectra of the reference and sample signals. (c) Extracted permittivity of the sample, shown together with the permittivity of undoped $4 \mathrm{H}-\mathrm{SiC}$ [12]. (d) The extracted conductivity of charge carriers in the co-doped $4 \mathrm{H}-\mathrm{SiC}$ sample. The full, black curves represent the best fit to the data by a Drude-Lorentz model, and the shaded areas indicate the contributions from the Drude and Lorentz terms, respectively. Light blue and red shadings indicate the real and imaginary parts of the contributions, respectively. (e) Our measured optical absorption in the visible range (blue, shaded curve), shown together with the structure of the five lowest conduction bands (solid curves) and density of states (shaded, red curve) (band structure adapted from Ref. [16]), and the location of the dopant energy levels (horizontal, shaded bars).

$345 \times 221 \mathrm{~mm}(300 \times 300$ DPI $)$ 


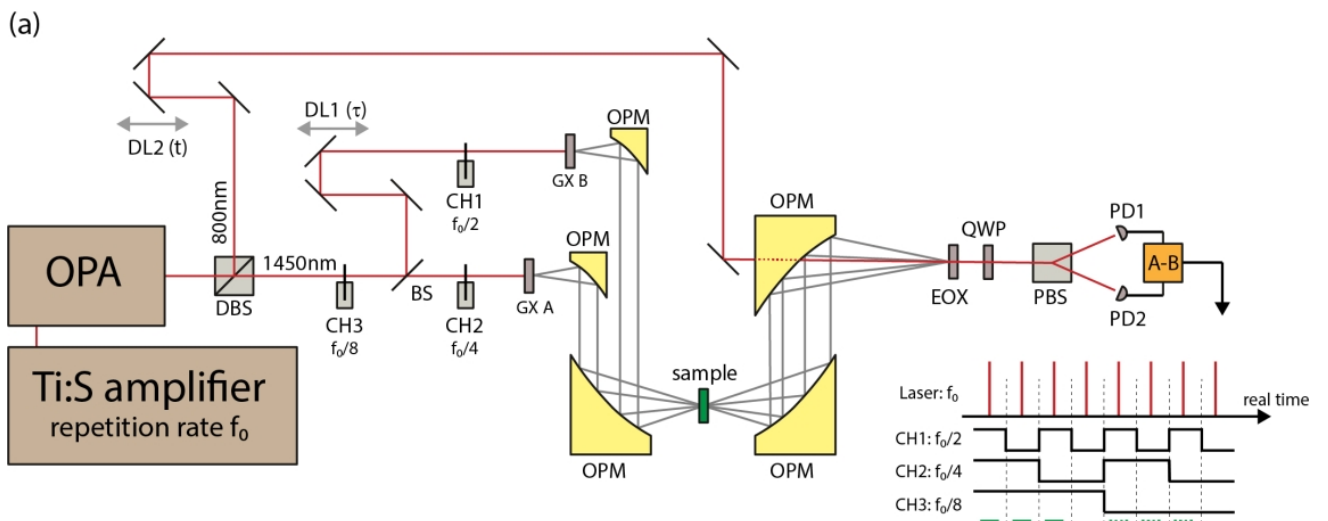

(b)

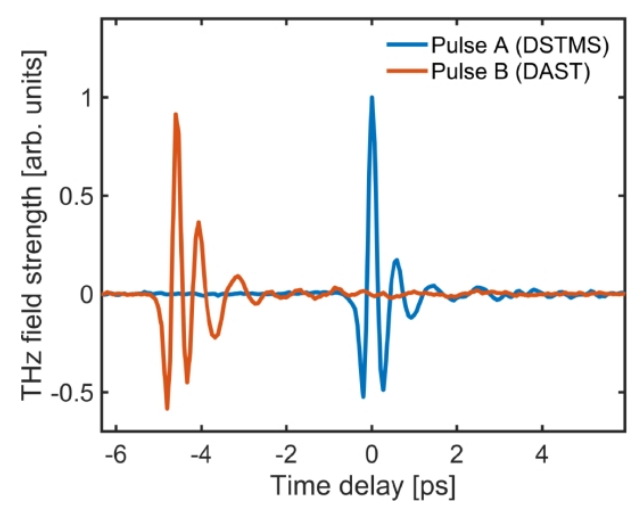

(c)

$B G$
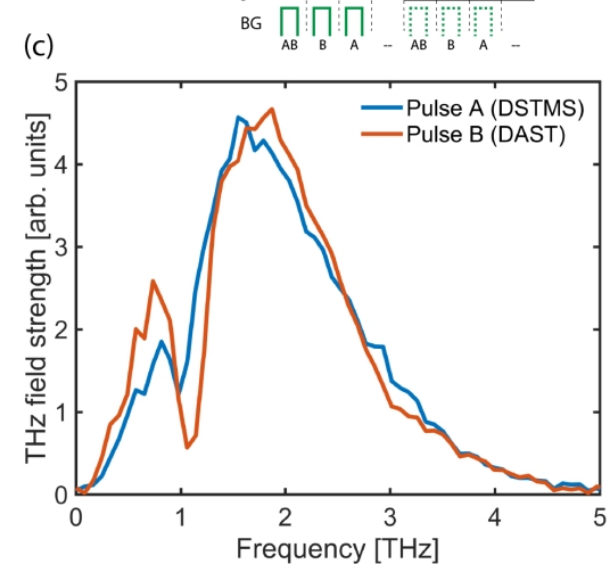

Schematic diagram of experimental setup for nonlinear 2D THz spectroscopy. Two THz pulses are generated in independent crystals (GX A and GX B), focused and overlapped at the sample position, and guided to electro-optic detection. A chopper scheme allows simultaneous recording pulse $A$ only, pulse $B$ only, and pulse $A$ and $B$ together. Pulse $A$ and $B$ are separated by a variable delay $T$. (b) Representative time traces of pulse $A$ and pulse B. (c) Frequency spectra of the two pulses.

$178 \times 146 \mathrm{~mm}(300 \times 300 \mathrm{DPI})$ 

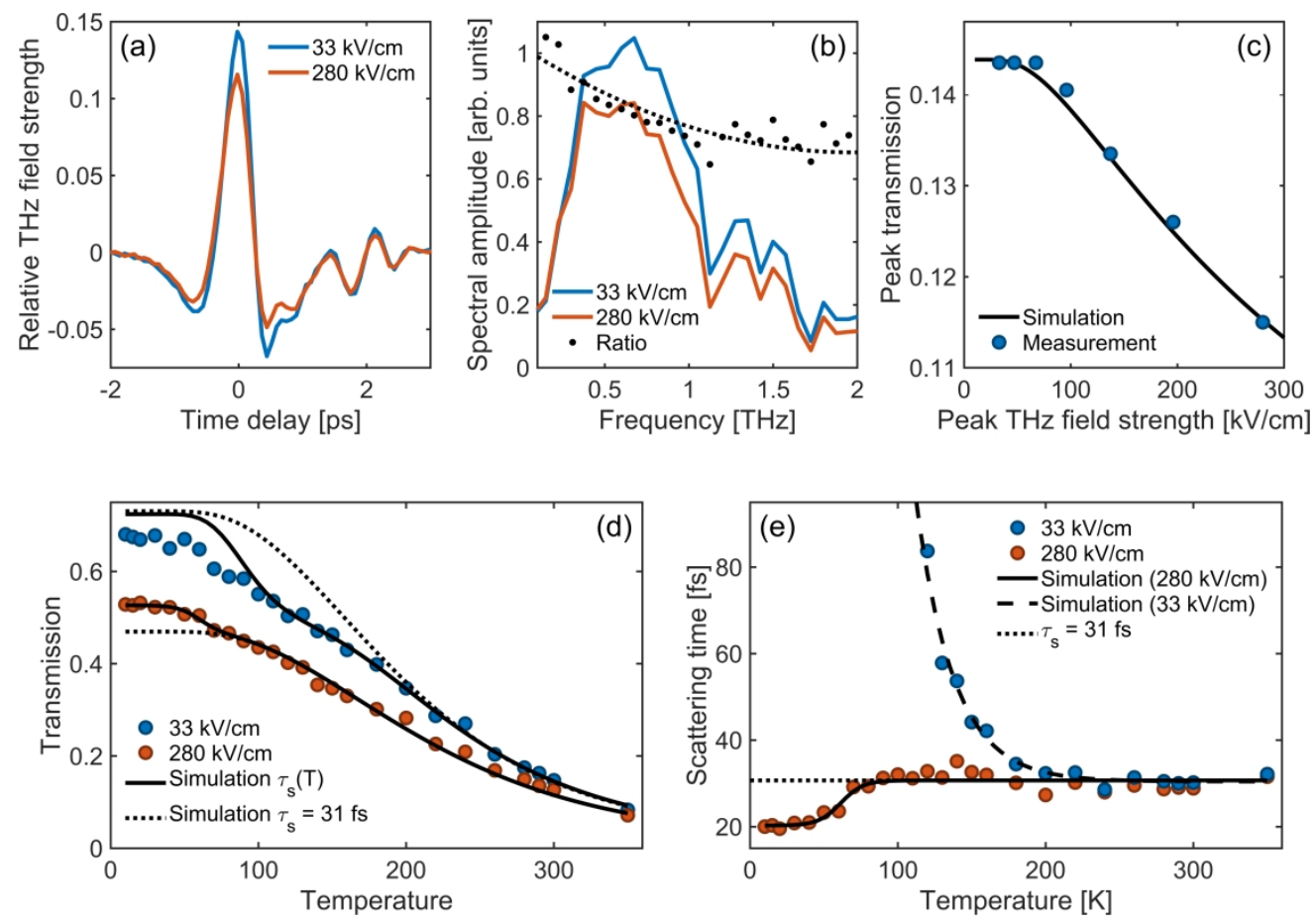

(a) Time traces of transmitted $\mathrm{THz}$ signals at 33 and $280 \mathrm{kV} / \mathrm{cm}$ peak field strength. The nonlinear response of SiC leads to decreased transmission. (b) Corresponding frequency spectra and ratio of the spectra (black data points and averaged, dashed curve). (c) Transmission through the SiC sample as function of peak incident field strength. Blue symbols are measured values, black curve is the nonlinear FDTD simulation result. (d) Temperature-dependent transmission at 33 and $280 \mathrm{kV} / \mathrm{cm}$ peak field strength, with nonlinear FDTD simulation results using temperature- and field-dependent scattering time (solid curves) and constant scattering time (dashed curves). (e) Temperature-dependent scattering time extracted from nonlinear FDTD simulations.

$337 \times 235 \mathrm{~mm}(300 \times 300 \mathrm{DPI})$ 

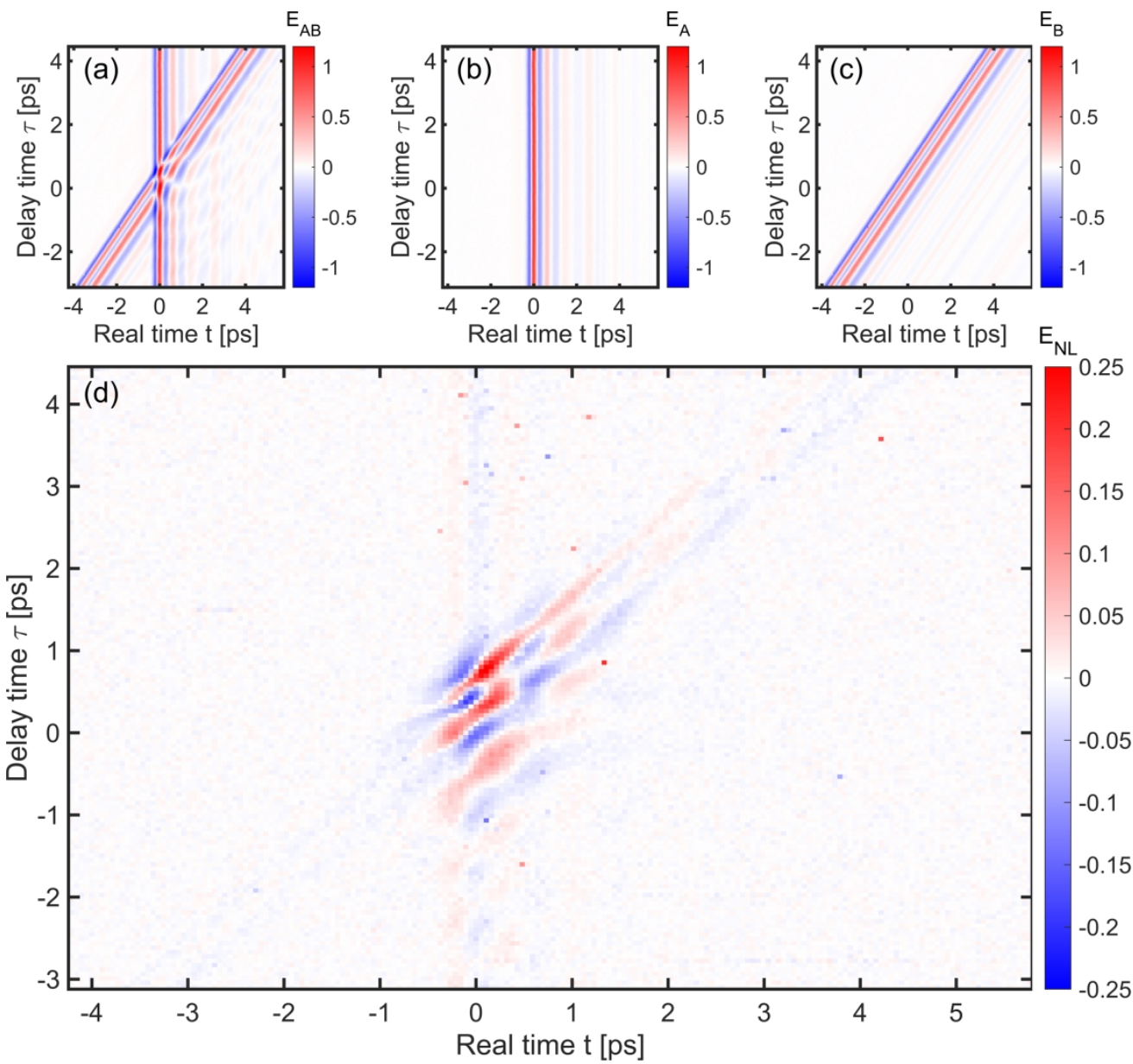

Time-domain representation of nonlinear two-dimensional THz spectroscopy of the SiC sample. (a) Two THz pulses $(A$ and $B$ ) interfering in the sample as function of the delay time between $A$ and $B$, (b) pulse $A$ alone, (c) pulse $B$ alone, and (d) the nonlinear response, defined as $E_{N L}(t, T)=E_{A B}(t, T)-E_{A}(t, T)-E_{B}(t, T)$.

$245 \times 229 \mathrm{~mm}(300 \times 300$ DPI $)$ 


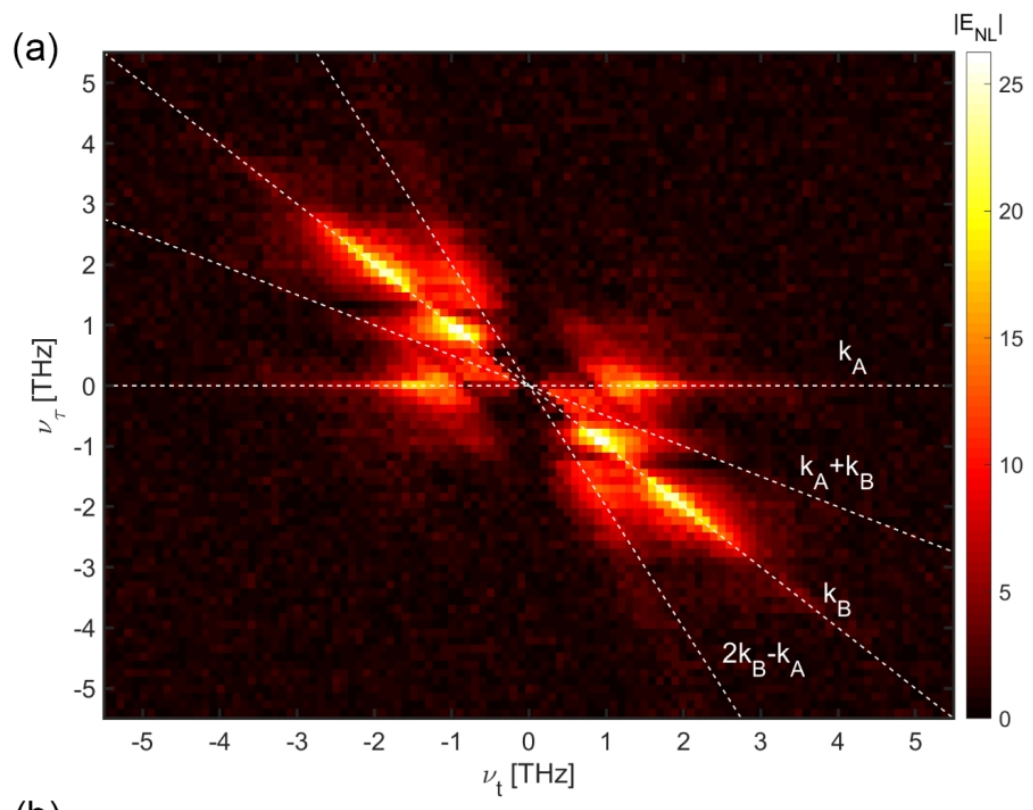

(b)

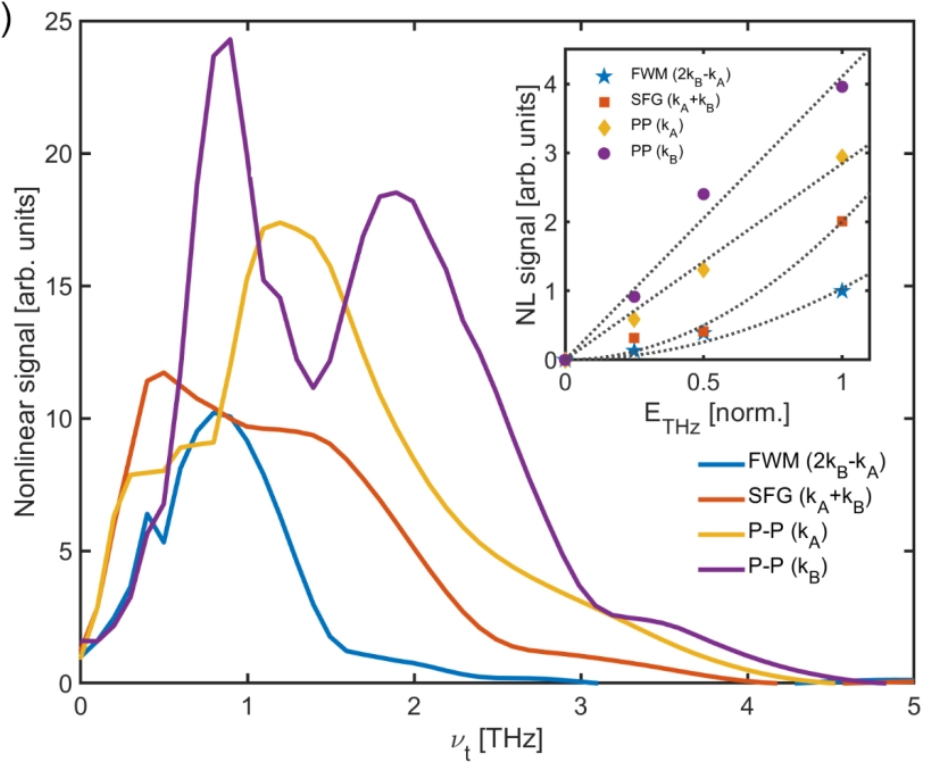

(a) Nonlinear 2D frequency spectrum of the nonlinear time-domain data shown in Figure 4, defined as $\left|E_{N L}\left(v_{t}, v_{T}\right)\right|=\left|E_{A B}\left(v_{t}, v_{T}\right)-E_{A}\left(v_{t}, v_{T}\right)-E_{B}\left(v_{t}, v_{T}\right)\right|$. Dashed lines indicate the expected positions of the pumpprobe signals $\left(k_{\mathrm{A}}\right.$ and $\left.k_{\mathrm{B}}\right)$, sum frequency generation $\left(k_{\mathrm{A}}+k_{\mathrm{B}}\right)$, and four-wave mixing $\left(2 k_{\mathrm{B}}-k_{\mathrm{A}}\right)$. (b) Line plots of the nonlinear signal along the indicated directions in (a). The inset shows the field dependence of the nonlinear signal contributions. Dashed curves are linear (PP) and quadratic (FWM, SFG) fits.

$114 \times 170 \mathrm{~mm}(300 \times 300 \mathrm{DPI})$ 